\title{
Homology-based repair induced by CRISPR- Cas nucleases in mammalian embryo genome editing
}

\author{
Xiya Zhang ${ }^{1}$, Tao $\mathrm{Li}^{1}$, Jianping Ou ${ }^{1 凶}$, Junjiu Huang ${ }^{2,3 \bowtie}$ (1) Puping Liang ${ }^{2,3 \bowtie}$ \\ ${ }^{1}$ Center for Reproductive Medicine, the Third Affiliated Hospital of Sun Yat-sen University, Sun Yat-sen University, Guangzhou \\ 510630, China \\ ${ }^{2}$ MOE Key Laboratory of Gene Function and Regulation, State Key Laboratory of Biocontrol, School of Life Sciences, Sun Yat- \\ sen University, Guangzhou 510275, China \\ ${ }^{3}$ Key Laboratory of Reproductive Medicine of Guangdong Province, the First Affiliated Hospital and School of Life Sciences, \\ Sun Yat-sen University, Guangzhou 510275, China \\ $\bowtie$ Correspondence: oujp3@mail.sysu.edu.cn (J. Ou), hjunjiu@mail.sysu.edu.cn (J. Huang), liangpp5@mail.sysu.edu.cn (P. \\ Liang)
}

Received November 8, 2020 Accepted March 16, 2021

\begin{abstract}
Recent advances in genome editing, especially CRISPRCas nucleases, have revolutionized both laboratory research and clinical therapeutics. CRISPR-Cas nucleases, together with the DNA damage repair pathway in cells, enable both genetic diversification by classical non-homologous end joining (c-NHEJ) and precise genome modification by homology-based repair (HBR). Genome editing in zygotes is a convenient way to edit the germline, paving the way for animal disease model generation, as well as human embryo genome editing therapy for some life-threatening and incurable diseases. HBR efficiency is highly dependent on the DNA donor that is utilized as a repair template. Here, we review recent progress in improving CRISPR-Cas nuclease-induced HBR in mammalian embryos by designing a suitable DNA donor. Moreover, we want to provide a guide for producing animal disease models and correcting genetic mutations through CRISPR-Cas nuclease-induced HBR in mammalian embryos. Finally, we discuss recent developments in precise genomemodification technology based on the CRISPR-Cas system.
\end{abstract}

Supplementary Information The online version of this article (https://doi.org/10.1007/s13238-021-00838-7) contains supplementary material, which is available to authorized users.
KEYWORDS homology-based repair (HBR), genome editing, disease modeling, embryo, precision medicine

\section{INTRODUCTION}

Genome editing, capable of rewriting DNA sequences in situ, holds tremendous potential in research and clinical applications. Genome editing in animal embryos provides an efficient way to generate genome-modified mammalian animals (e.g., mice, rats, rabbits, pigs, and monkeys), which holds tremendous potential in disease modeling and precision medicine (Anzalone et al., 2020; Doudna, 2020). Furthermore, genome editing in human embryos may be an option to prevent genetic disease transmission and save lives once the technical, safety, ethical, social, and legal issues associated with human embryo genome editing have been resolved (Baltimore et al., 2015; Rossant, 2018; Macintosh, 2019).

Since the first report of genome editing by meganuclease in the 1990s (Rouet et al., 1994; Choulika et al., 1995), zinc finger nuclease (ZFN), transcription activator-like effector nuclease (TALEN), and clustered regularly interspaced short palindromic repeat (CRISPR)-CRISPR-associated (CRISPR-Cas) nucleases have been utilized for genome editing (Urnov et al., 2010; Gaj et al., 2013). Among these four kinds of genome editing tools, CRISPR-Cas nucleases stand out as the most convenient, cost-effective, versatile, and robust tool (Anzalone et al., 2020). CRISPR-Cas nuclease (e.g., Cas9, Cas12a, Cas12b, and CasX), adapted 
from the adaptive immune system of bacteria and archaea, is an RNA-protein complex composed of guide RNA (gRNA) and Cas protein (Jinek et al., 2012; Cong et al., 2013; Mali et al., 2013). The gRNA-Cas complex searches for the target DNA containing both the protospacer adjacent motif (PAM) sequence and the sequence complementary to the gRNA (Sternberg et al., 2015; Jiang et al., 2016; Palermo et al., 2016, 2017; Chen et al., 2017). The guide sequence at the end of the gRNA pairs with the complementary strand (or named target strand) of the target DNA, resulting in CRISPR-Cas nuclease activation and DNA double-stranded breaks (DSBs) (Sternberg et al., 2015; Jiang et al., 2016; Palermo et al., 2016, 2017; Chen et al., 2017). DNA DSBs generated by CRISPR-Cas nuclease can be repaired by either classical non-homologous end joining (c-NHEJ) or homology-directed repair (HBR) (Fig. 1) (Cong et al., 2013; Mali et al., 2013). c-NHEJ will result in random small deletions or insertions at the target site. In addition, in the presence of an exogenous DNA donor, c-NHEJ may lead to DNA donor integration at the target site without homology arms (homology-independent targeted integration, HITI) (Fig. 1) (Suzuki et al., 2016). However, HITI tends to induce mutations at the junctions between the DNA donor and the target site (Suzuki et al., 2016). Provided with single-stranded oligodeoxynucleotides (sSODN), whose homology arm could pair with both the DNA donor and the target site, further increased the targeted integration efficiency of HITI (Yoshimi et al., 2016). HBR includes homology-directed repair (HDR), microhomology-mediated end joining (MMEJ, or alternative end-joining), and single-stranded annealing (SSA) (Bennardo et al., 2008; Ceccaldi et al., 2016; Chang et al., 2017). HDR can be further divided into single-stranded templated repair (SSTR) and homologous recombination (HR), depending on the donor DNA type (Fig. 1) (Sakuma and Yamamoto, 2017; Richardson et al., 2018; Yeh et al., 2019). HBR can utilize exogenous DNA donors with homology arms to repair DSB, resulting in the precisely edited target genes, including precise deletion, precise point mutation, tag (or reporter gene) integration, and conditional allele generation (Yang et al., 2013). To prevent re-cutting of the edited allele, the exogenous DNA donor should contain both the desired sequence change and blocking silent mutations, which will prevent the gRNA-Cas complex from cleaving the edited allele (Paquet et al., 2016; Kwart et al., 2017). It is noteworthy that MMEJ and SSA can also occur between two homologous sequences flanking the target site, deleting the intervening sequence (Shen et al., 2018; Zhang and Matlashewski, 2019). Although recently developed base editors and prime editors could realize some of the functions (e.g., precise C-to-T conversion, precise A-to-G conversion, precise C-to-G conversion, small DNA fragment deletion, and small DNA fragment insertion) of CRISPR-Cas nucleaseinduced HBR, they could not be utilized to catalyze other types of base conversion (e.g., G-to-C conversion), inserting large DNA fragments, and generating a conditional allele (Komor et al., 2016; Gaudelli et al., 2017; Anzalone et al.,
Figure 1. DNA repair pathways involved in CRISPRCas nucleases mediated genome editing in mammalian cells. CRISPR-Cas nuclease-induced DNA DSBs are repaired by either C-NHEJ or homology-based repair (HBR). The recruitment of 53BP1 inhibits end resection at the DSB site, promoting DSB repair via the c-NHEJ pathway. Without exogenous DNA donors, the two ends of the DSB are ligated together precisely or imprecisely with 1-4 bp small insertion or deletion (indel) through the c-NHEJ pathway. In the presence of a double-stranded DNA (dsDNA) donor without homology arms, the dsDNA donor can be inserted into the DSB site via the c-NHEJ pathway (HITI). In addition, the two ends of the DSB site may undergo initial phase end resection by RBBP8 (or named CtIP) and MRN, generating short $3^{\prime}$ overhang. Supplied with a DNA donor with homology arms, the $3^{\prime}$ overhang will prime DNA repair through the high-fidelity homology-based repair pathway. Supplied with a dsDNA donor with short homology arms (5-40 bp), the DSB site could be repaired via the MMEJ pathway. Furthermore, the short $3^{\prime}$ overhang may undergo second phase end resection, generating a longer $3^{\prime}$ overhang. In the presence of dsDNA donor with medium homology arms (>200 bp), the DSB site could be repaired via the SSA pathway. Supplied with a dsDNA donor with long homology arms ( $\geq 400 \mathrm{bp}$ ), the DSB site could be repaired via the HR pathway. In the presence of ssDNA donor with short homology arms ( $\geq 30 \mathrm{nt}$ ), the DSB site could be repaired via the SSTR pathway. Some major proteins involved in each pathway are shown in the figure. The direction of the arrow represents the $5^{\prime}$ to $3^{\prime}$ direction. Deletion, black cross. Inserted fragment, colored line. c-NHEJ, classical nonhomologous end joining. HITI, homology-independent targeted integration. MRN, MRE11-RAD50-NBS1 complex. HA, homology arm. SSTR, single-stranded templated repair. HR, homologous recombination. SSA, singlestranded annealing. MMEJ, microhomology-mediated end joining.

2019; Kurt et al., 2020; Zhao et al., 2020a). However, HBR is much less efficient than c-NHEJ in mammalian embryos, resulting in mosaicism in edited embryos. Mosaic embryos contain precisely edited cells, imperfectly edited cells, and non-edited cells. For small mammalian animals, such as mice, rats, and rabbits, homozygous edited animals could be generated by repeated breeding. However, repeated breeding is a bottleneck for large mammalian animal model generation, which requires a longer time to reach sexual maturity (e.g., pigs and monkeys). Moreover, mosaicism and unintended editing are the main technical issues that impede the clinical application of human embryo genome editing therapy (Zuccaro et al., 2020). Improving CRISPR-Cas nuclease-induced HBR efficiency will mitigate mosaicism in embryo genome editing (Zuccaro et al., 2020). Taken 


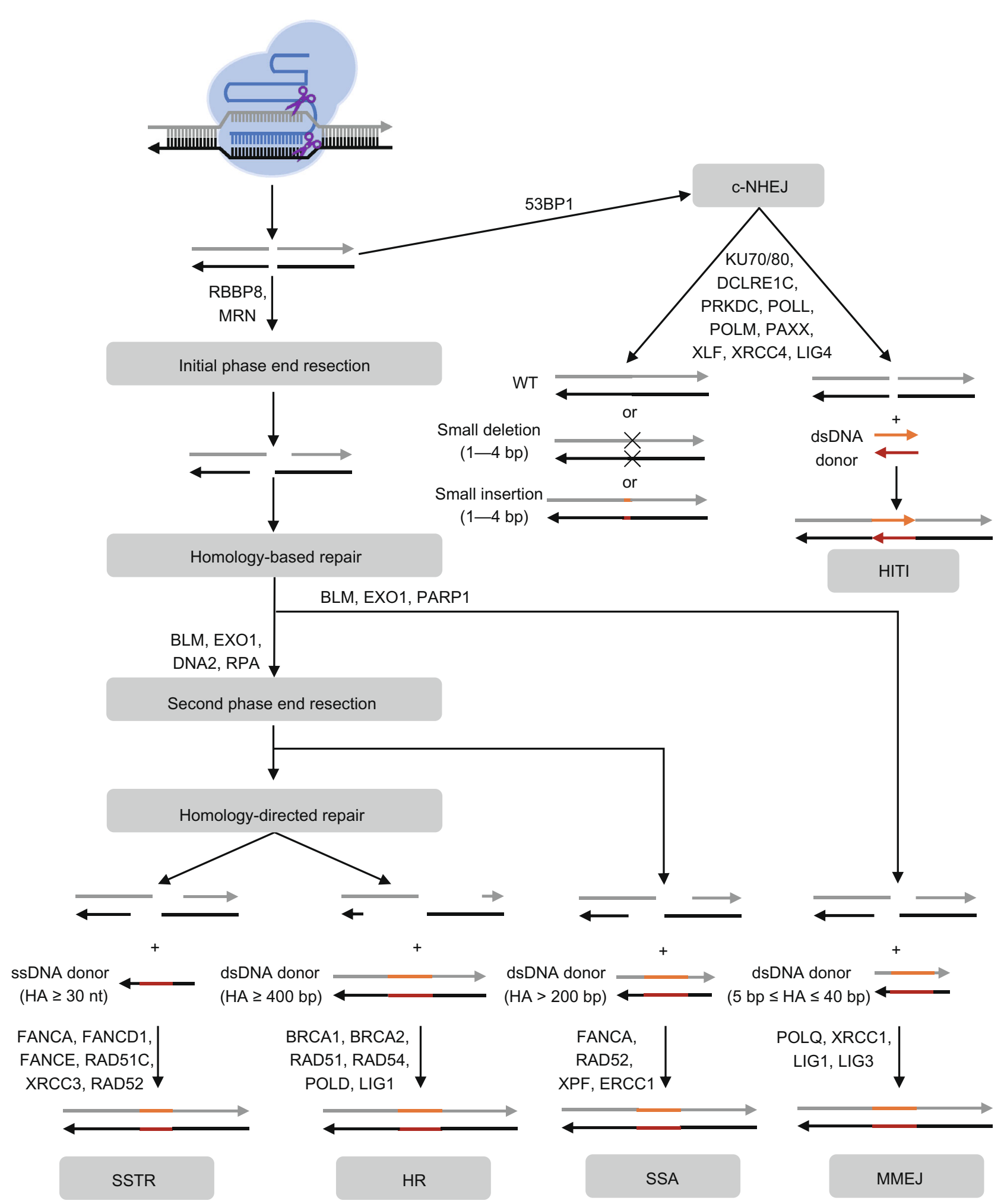


together, it is of great significance to develop new methodologies to improve HBR efficiency in mammalian embryos.

There have already been excellent reviews on recent advances in improving HBR repair by controlling DNA repair pathways (Liu et al., 2018a; Yeh et al., 2019). The DNA donor is a critical determinant of HBR efficiency. In this review, we restrict our discussion to the topic of improving HBR by designing suitable DNA donors for genome editing in mammalian embryos, especially in frequently investigated rodent embryos. There are several types of DNA donors, including single-stranded DNA (ssDNA), double-stranded plasmid, and linear double-stranded DNA (dsDNA). Genome editing outcomes in embryos are believed to be similar between mice and large mammalian animals. Comparative analysis of these data will provide a practical guide for embryo gene editing in large mammalian embryos (Ma et al., 2017a, 2018; Adikusuma et al., 2018; Wilde et al., 2018; Zuccaro et al., 2020).

\section{HOMOLOGY-BASED REPAIR USING SINGLE- STRANDED DNA DONOR}

Single-stranded DNA (ssDNA) donors include ssODN, long single-stranded DNA (IssDNA), and single-stranded adenoassociated virus (AAV) genomic DNA. ssODN, which is typically no longer than $200 \mathrm{nt}$, could be synthesized by a commercial company. IssDNA is an ssDNA longer than 200 nt and could be generated by in vitro transcription and reverse transcription (ivTRT) (Miura et al., 2015; Quadros et al., 2017; Codner et al., 2018; Li et al., 2019), chemical synthesis (Quadros et al., 2017), selection of ssDNA labeled with biotin (Stahl et al., 1988), and the strand-specific digestion of dsDNA (Murgha et al., 2014; Yoshimi et al., 2016). AAV is a single-stranded DNA virus with a genome of approximately $4.8 \mathrm{~kb}$. AAV could be produced by transfecting HEK-293T cells with double-stranded AAV plasmid vector, Rep-Cap plasmid, and helper plasmid (Chen et al., 2020). Upon AAV infection, single-stranded AAV genomic DNA is released into the cell and works as a repair template (Yoon et al., 2018; Chen et al., 2019).

Compared with double-stranded DNA (dsDNA) donors, such as plasmids and linear dsDNA, the knock-in (KI) efficiency using single-stranded DNA (ssDNA) donor is much higher (Miura et al., 2015; Codner et al., 2018). Owing to the SSTR pathway utilized by ssDNA donors, the homology arm of SSDNA donors is much shorter than plasmid and linear dsDNA, which enables the convenient and high-throughput construction of DNA donors (Kan et al., 2017). These two features make ssDNA an ideal DNA donor.

\section{HBR using ssODN}

sSODN could be used as a repair template to generate precise modifications, such as point mutations, small insertions, or precise deletions. To achieve high editing efficiency, the distance between the modification and the Cas nuclease
Figure 2. Conditional allele generation strategies in mammalian embryos. Four different strategies, indicated with solid lines, have been exploited to generate conditional allele depending on the distance between the two gRNA cleavage sites (defined as $X$ ). If $X \leq 1,351 \mathrm{bp}$, a IssDNA donor is recommended. If $X>1,351 \mathrm{bp}$, a dsDNA donor is recommended. The dashed line indicates two possible strategies, namely HMEJ and Tild, which remain to be tested in mammalian embryos. The upper limit of the conditional allele ( $n \mathrm{bp}$ ) that could be generated using a dsDNA donor remains to be investigated. Generating conditional allele using ssODN is inefficient, however, it might be used to generate very large conditional alleles $(\mathrm{X}>\mathrm{n}$ bp). Intron, black line. Exon, blue box. gRNA cleavage site, yellow lightning bolt. LoxP, red triangle. Plasmid backbone, blue line. IssDNA, long single-stranded DNA. SSTR, single-stranded templated repair. dsDNA, double-stranded DNA. HR, homologous recombination. HMEJ, homology-mediated end joining. Tild, targeted integration with linearized dsDNA. PITCh, precise integration into the target chromosome. ssODN, single-stranded oligodeoxynucleotide.

cleavage site should be as small as possible ( $\leq 30 \mathrm{bp}$ ) ( $\mathrm{Re}-$ naud et al., 2016; Quadros et al., 2017). Generally, ssODN contains a $\geq 30$-nt homology arm at both the $5^{\prime}$ and $3^{\prime}$ end, where a longer homology arm could increase the editing efficiency (Renaud et al., 2016). However, ssODN with a longer homology arm also displays more severe cytotoxicity (Okamoto et al., 2019).

Previous genome editing studies using ZFN and TALEN have proven that SSODN could be used as a DNA donor to generate precisely edited cells and animal models (Chen et al., 2011; Bedell et al., 2012; Shen et al., 2013). Combing Cas9 nuclease with ssODN enables the efficient insertion of a restriction enzyme recognition site in human cells (Cong et al., 2013; Ran et al., 2013). Delivering Cas9 nuclease together with sSODN has previously led to the efficient correction of $H B B$ and $C Y B B$ mutations in human hematopoietic stem and progenitor cells (HSPCs) (DeWitt et al., 2016; De Ravin et al., 2017). It is reported phosphorothioate (PS) bound at the several terminal nucleotides of sSODN could improve targeted insertion efficiency by protecting the ssODN from degradation (Papaioannou et al., 2009), and was previously found to improve the repair efficiency of the CYBB mutation in HSPCs (De Ravin et al., 2017). However, the repair efficiency of $H B B$ mutations was not improved by phosphorothioate (PS) modification (DeWitt et al., 2016). Thus, whether phosphorothioate (PS) modification improves the targeted insertion efficiency is likely to depend on the ssODN used. In addition to phosphorothioate (PS) modification, locked nucleic acid (LNA) modification could also 


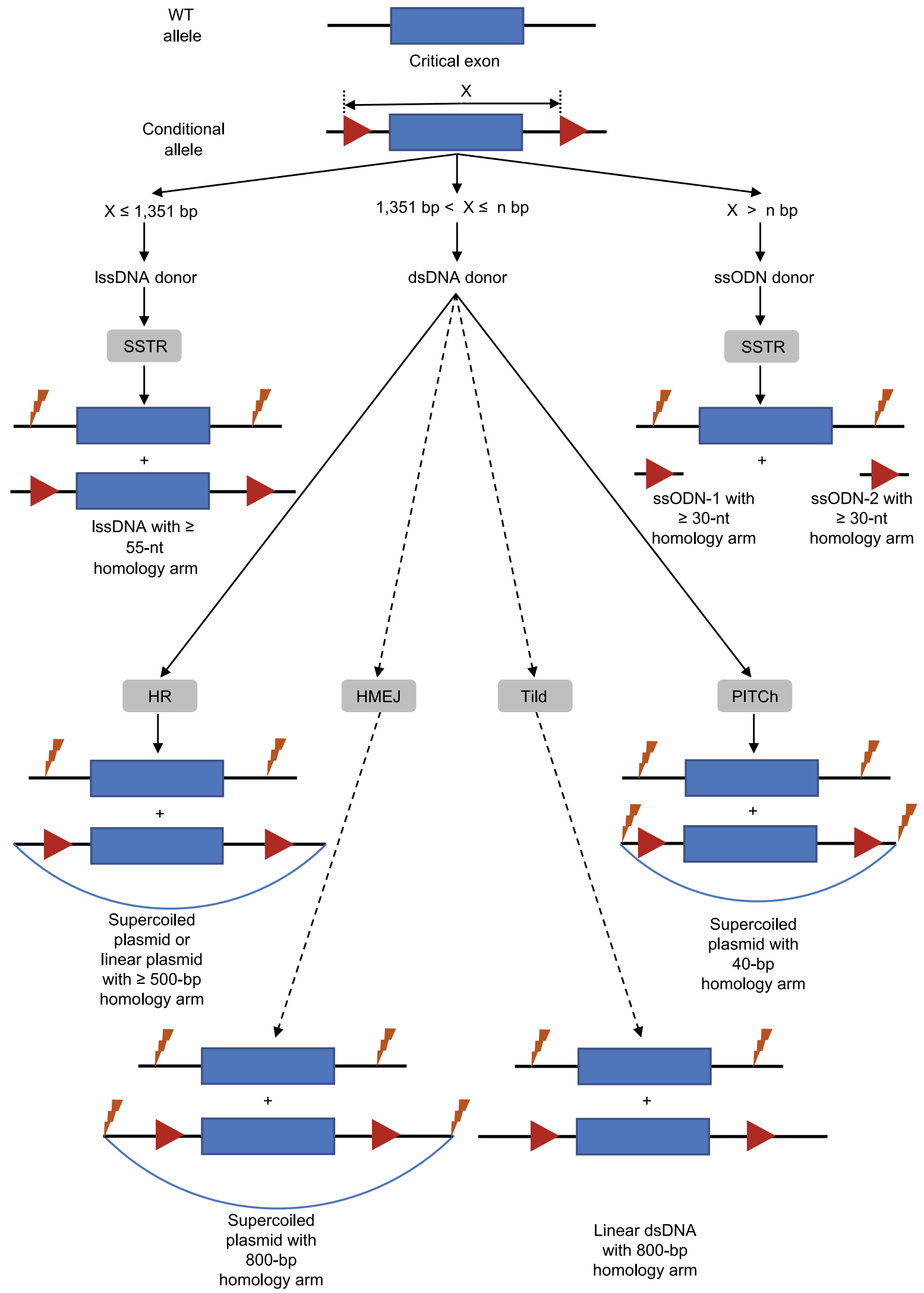


enhance $\mathrm{KI}$ efficiency in human cells by improving the stability of ssODN (Renaud et al., 2016).

Co-injecting SSODN together with CRISPR-Cas nuclease into zygotes leads to highly efficient $\mathrm{KI}$ of small fragments in mouse, rat, rabbit, pig, sheep, bovine, and even human embryos (Table S1). In line with the cellular data, PS-modified SSODN also showed improved $\mathrm{KI}$ efficiency in mouse and rat embryos (Table S1) (Renaud et al., 2016). However, whether PS- or LNA-modified ssODN improves the $\mathrm{KI}$ efficiency in large mammalian animal and human embryos remains to be investigated.

One limitation of sSODN is its limited length ( 200 nt), making it impossible to achieve larger DNA fragment $\mathrm{KI}$. Although using multiple ssODNs with overlapping regions allows larger fragment $\mathrm{KI}$ in $C$. elegans, overlapping ssODNs generated incomplete $\mathrm{KI}$ in rats (Paix et al., 2016; Remy et al., 2017). Whether ssODNs with overlapping regions could result in larger DNA fragment $\mathrm{KI}$ in the emfbryos of large mammalian animals and humans remains unclear.

Although there are some cases that used two gRNAs and two ssODNs containing the LoxP sequence to generate conditional allele (referred herein as two-ssODN floxing method) (Fig. 2), generating a conditional allele using ssODN is inefficient (Yang et al., 2013; Pritchard et al., 2017; Lanza et al., 2018; Gurumurthy et al., 2019). In one comprehensive study, 56 genes were selected for generating the conditional allele by the two-ssODN floxing method (Gurumurthy et al., 2019). Among the 1,718 mouse pups generated, only $15(0.87 \%)$ pups harbored the conditional allele (Gurumurthy et al., 2019). One major problem is that the two LoxP sites are integrated in trans, which means that they integrate into two different chromosomes instead of the same chromosome (Lanza et al., 2018; Gurumurthy et al., 2019). However, one advantage of the two-ssODN floxing method is that the distance between gRNA target sites (250$4,500 \mathrm{bp}$ ) did not affect the frequency of conditional allele generation (Lanza et al., 2018). This advantage is beneficial for generating a large conditional allele, making it feasible to knock out very large DNA fragment upon induction.

\section{HBR using IssDNA}

The maximum length of IssDNA is 5,000 nt, after which they are prone to spontaneous breaks (Lanza et al., 2018). Compared with ssODN, IssDNA allows for larger fragment knock-ins and the efficient generation of conditional alleles in mouse and rat zygotes (Fig. 2 and Table S2) (Miura et al., 2015; Yoshimi et al., 2016). IssDNA uses a short homology arm (typically 55-329 nt at each end) to $\mathrm{KI}$ exogenous DNA (Table S2). Similar to ssODN, the distance between the modification and the Cas nuclease cleavage site should be as small as possible ( $\mathrm{Li}$ et al., 2019). Combining two gRNAs and IssDNA, the intended mutation located between the two gRNA cleavage sites, which is remote from both gRNA cleavage sites (>30 bp), could be installed with a high efficiency (Table S2) (Codner et al., 2018). Utilizing IssDNA,
Codner et al. generated a point-mutation mouse model whose intended mutation was 98-bp away from the gRNA cleavage site (Codner et al., 2018). However, one drawback of IssDNA is that it is more toxic than SsODN (Li et al., 2019).

IssDNA has been exploited for large fragment $\mathrm{KI}$ in cells and animal embryos, but not in human embryos (Miura et al., 2015; Yoshimi et al., 2016; Li et al., 2019). Using IssDNA as a donor results in a high GFP $\mathrm{KI}$ efficiency in human HEK$293 \mathrm{~T}$ cells $(5 \%-30 \%)$. Furthermore, longer homology arms improved the KI efficiency in HEK-293T cells, with 400-700 nt at each end as the optimal homology arm length ( $\mathrm{Li}$ et al., 2019). In mouse zygotes, Rolen et al. were able to knock in 1,368-bp DNA fragment using IssDNA with a 90-nt homology arm at each end (Table S2) (Quadros et al., 2017). However, it remains unclear whether it is possible to generate larger DNA fragment $\mathrm{KI}$ (e.g., 5,000-nt DNA fragment) in animal zygotes using short homology arms (55-144 nt). To improve the efficiency of large DNA fragments $\mathrm{KI}$, IssDNA with longer homology arms may be helpful (Li et al., 2019). PS-modifed IssDNA might also be able to improve the HBR efficiency, but it has not yet been tested in cells and mammalian embryos.

In addition, IssDNA can be used to generate a conditional allele. To the best of our knowledge, IssDNA has been successfully used to generate a 1,351-bp conditional allele (Table S2) (Codner et al., 2018). However, whether it is feasible to generate a larger conditional allele using IssDNA is unclear (Codner et al., 2018). Compared with ssODN, generating a conditional allele using IssDNA is much more efficient (Tables S1 and S2) (Codner et al., 2018; Gurumurthy et al., 2019). Because IssDNA harbors two LoxP sites, it requires only one recombination event, whereas the two-ssODN floxing method requires two simultaneous recombinations in the same chromosome (Quadros et al., 2017; Codner et al., 2018; Gurumurthy et al., 2019). Thus, IssDNA alleviates the challenge of integration in trans and improves the efficiency of generating a conditional allele. However, longer homology arms (100 nt vs. $60 \mathrm{nt})$ may be another important factor to enhance the efficiency (Tables S1 and S2) (Quadros et al., 2017).

However, producing IssDNA is more expensive and cumbersome than ssODN. The chemical synthesis of IssDNA is costly. The ivTRT method can generate a large amount of $\leq 2,000-n t$ IssDNA (>50 $\mu \mathrm{g}$ ) (Li et al., 2019). However, the generated IssDNA will be contaminated by truncated IssDNA due to the poor processivity of the reversetranscriptase (Mohr et al., 2013). The selection of ssDNA labeled with biotin and the strand-specific digestion of dsDNA require generating dsDNA by PCR, which may introduce sequence error ( $\mathrm{Li}$ et al., 2019). Although the strand-specific digestion of dsDNA is more convenient than ivTRT, the amount of IssDNA generated is approximately 10-fold lower than that of the ivTRT method (Murgha et al., 2014; Yoshimi et al., 2016; Li et al., 2019). In addition, all the methods used to produce IssDNA may result in unexpected mutations in the DNA donor. Therefore, it is necessary to 
sequence the edited cells and animals to eliminate the effect of unexpected mutations.

\section{HBR using AAV DNA}

In addition to ssODN and IssDNA, the linear single-stranded genomic DNA of AAV has been utilized as HBR templates. $A A V$ is a single-stranded DNA virus that can be produced by transfecting virus-packaging cells with double-stranded AAV plasmids vectors.

Combining ZFN (or TALEN) with AAV serotype 6 (AAV6) donor, which contains homology arms flanking the insertion sequence, efficient targeted insertion could be induced in HSPCs and primary T cells (Sather et al., 2015; Wang et al., 2015). Later, it has been proven that combing CRISPR-Cas nuclease with AAV6 donor vector could also induce efficient KI in HSPCs (Dever et al., 2016; Pavel-Dinu et al., 2019). Intriguingly, the AAV serotype 1 (AAV1) donor vector induced a higher $\mathrm{KI}$ efficiency than conventional plasmid donor vector when combined with CRISPR-Cas nuclease in HEK-293T cells, U2OS cells, human dermal fibroblasts, and rat $\mathrm{C} 6$ cells (Gaj et al., 2017). Furthermore, the AAV donor is delivered to the liver, together with CRISPR-Cas nuclease, efficiently correcting the mutation in the hereditary tyrosinemia mouse model and hyperammonemia mouse model (Yang et al., 2016; Yin et al., 2016; Krooss et al., 2020). Delivering AAV donor and CRISPR-Cas nuclease into mouse zygotes results in efficient genome editing, including precise point mutation and $\mathrm{KI}$ of large DNA fragments (771-3,300 nt) (Table S3) (Yoon et al., 2018; Chen et al., 2019). However, whether it is feasible to generate a conditional allele using an AAV donor remains to be investigated.

One constraint of the AAV donor is its limited packaging capacity $(\sim 4,600 \mathrm{nt})$, making it difficult to insert larger gene coding sequences (e.g., DMD and F8) (Bak and Porteus, 2017). In general, the homology arm length of the AAV donor vector is $400-800 \mathrm{nt}$. Considering the two flanking 400-nt homology arms, the length of the inserted DNA fragments should be shorter than 3,800 nt (Bak and Porteus, 2017). By delivering two AAV donors together with CRISPR-Cas nuclease, it is feasible to insert 5,700-nt DNA in HSPCs and T cells (Bak and Porteus, 2017). However, whether it is possible to edit target genes in large mammalian animal and human embryos using AAV donors remains to be tested (Chen et al., 2011; Gaj et al., 2017). First, the AAV serotype that could infect large mammalian animal and human zygotes should be screened. Then, the editing efficiency using the AAV donor vector should be investigated. It is worth noting that using an AAV donor vector may impose new safety concerns about AAV infection.

HBR using sSODN and IssDNA has been shown to occur through the SSTR pathway (Renaud et al., 2016; Kan et al., 2017; Richardson et al., 2018). However, whether HBR using an AAV donor also occurs through the SSTR pathway remains to be elucidated.
SSTR is similar to the synthesis-dependent strand annealing (SDSA) pathway of HR (Kan et al., 2017; Yeh et al., 2019). While the SSTR pathway takes advantage of single-stranded DNA donors (Kan et al., 2017), the HR pathway utilizes a double-stranded DNA (dsDNA) donor. Unlike HR, SSTR is not Rad51-dependent (Bothmer et al., 2017; Richardson et al., 2018). Contrary to MMEJ and SSA, which are both independent of templated DNA synthesis using exogenous DNA donor as the template, SSTR and HR are characterized by strand invasion and subsequent strand extension using exogenous DNA donor as the template (Kan et al., 2017). However, the proteins involved in SSTR remain under-explored. It has been proposed that some proteins responsible for the MMEJ, SSA, and Fanconi anemia pathway may be involved in SSTR (Quadros et al., 2017; Richardson et al., 2018). To identify the proteins involved in the SSTR pathway, systemic loss-of-function screening is needed (Richardson et al., 2018). Knocking down either Fanca or Fancd1, genes involved in the Fanconi anemia pathway, was found to inhibit AAV-mediated $\mathrm{KI}$ in mouse cardiomyocytes (Kohama et al., 2020), suggesting that AAVmediated $\mathrm{KI}$ occurs via the SSTR pathway.

It is noteworthy that genome editing using ssODN (or IssDNA) as HBR templates often displays deletion mutations at the $5^{\prime}$ end of the ssODN (or IssDNA) repair template (Renaud et al., 2016; Ge and Hunter, 2019). The 5'-end deletion may be due to the incomplete DNA synthesis and the MMEJ pathway-mediated deletion in cells and embryos (Fig. 3) (Renaud et al., 2016; Yoshimi et al., 2016). The elongation of the 5' homology arm ameliorated the 5'-end deletion and enhanced the $\mathrm{KI}$ efficiency of SSODN and IssDNA, indicating that asymmetric ssODN and IssDNA with longer 5' homology arm is better than symmetric ssODN (Renaud et al., 2016; Richardson et al., 2016; Yoshimi et al., 2016; Lanza et al., 2018; Wang et al., 2018). However, it remains to be investigated whether $\mathrm{KI}$ by $\mathrm{AAV}$ donor also shows a 5 ' deletion propensity, similar to sSODN and IssDNA, when partial KI occurs (Renaud et al., 2016; Yoshimi et al., 2016; Canaj et al., 2019). 5'-end deletion propensity is an important feature of the SSTR pathway. If it does show 5 '-end deletion propensity, $\mathrm{KI}$ by AAV donor might occur through SSTR pathway. In addition, whether elongating the 5' homology arm will also enhance the precise $\mathrm{KI}$ efficiency of the AAV donor remains to be investigated.

Because the non-target strand is released earlier than the target strand after DNA cleavage by Cas9, Cas9 nucleaseinduced HBR using ssODN donor showed donor strand bias at some sites (Richardson et al., 2016; Lanza et al., 2018). Combined with the Cas9 nuclease, ssODN complementary to the non-target strand displays higher HBR efficiency than ssODN, which is complementary to the target strand (Richardson et al., 2016; Lanza et al., 2018). In contrast to Cas9, Cas12a (e.g., AsCas12a and LbCas12a) exhibit a preference for ssODN complementary to the target strand (Wang et al., 2018). Whether the target strand is released earlier than the non-target strand after DNA cleavage by 


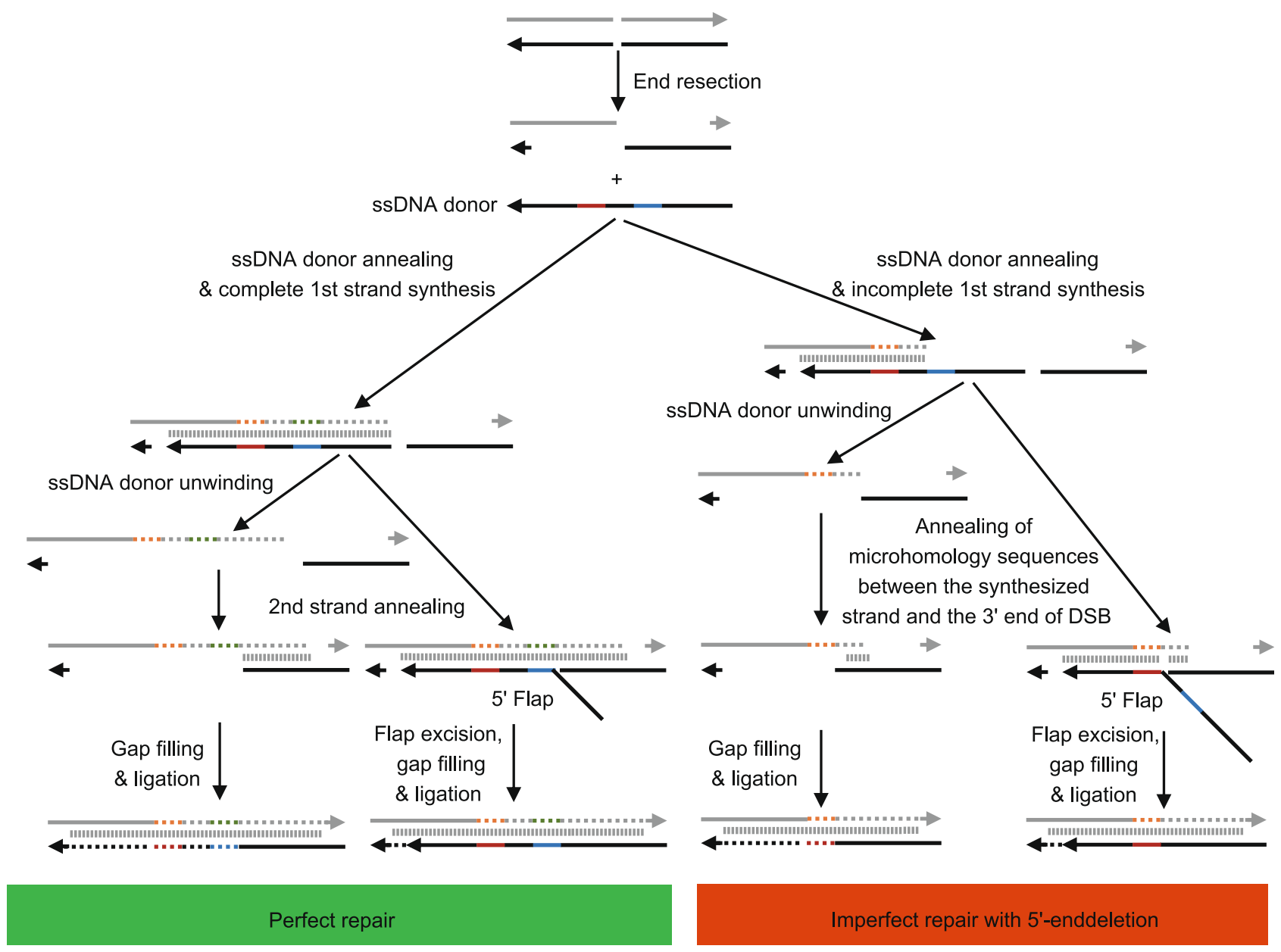

Figure 3. Potential mechanisms underlying perfect repair and imperfect repair via the SSTR pathway. DSB site, generated by CRISPR-Cas nuclease, undergoes end resection and generates 3' overhangs. In the presence of ssDNA donor, the 3' overhang anneals with the ssDNA donor and primes DNA synthesis, leading to complete or incomplete 1st strand synthesis. Complete 1st strand synthesis copies both the 3'-end edit (red line) and 5'-end edit (blue line). However, incomplete 1st strand synthesis only copies the 3 '-end edit (red line). After 1st strand synthesis, the ssDNA donor is removed (or not removed) by helicase. The newly synthesized 1st strand anneals with homology sequences at the other end of the DSB site. If the ssDNA donor is removed, the DSB is repaired by gap filling and ligation. If the ssDNA donor is not removed, the annealing process generates a repair intermediate with a $5^{\prime}$ flap, which will be further repaired by flap excision, gap filling, and ligation, resulting in the retention of ssDNA donor at the target site. Whether SSTR results in the retention of SSDNA donor at the target site is still under debate. During incomplete 1st strand synthesis, the newly synthesized 1st strand searches the DSB site for microhomology sequences for hybridization, resulting in an imperfect repair without a 5'-end edit. The direction of the arrow represents the 5 ' to $3^{\prime}$ direction. $3^{\prime}$-end edit, red line. 5'-end edit, blue line. Newly synthesized DNA, dashed line. SSTR, single-stranded templated repair. DSB, double-stranded break.

Cas12a remains to be investigated. In addition, whether CRISPR-Cas nuclease-induced HBR using IsSDNA and AAV donor vector also displays such strand bias remains unclear.

\section{HOMOLOGY-BASED REPAIR USING DOUBLE- STRANDED DNA DONOR}

dsDNA donors, including plasmid donor and linear dsDNA donor, are suitable for inserting large DNA fragments $(>3.8$ $\mathrm{kb}$ ), which is not possible using a single AAV vector (Table S4). HBR using dsDNA donors is less efficient than single-stranded DNA, but allows for larger fragment $\mathrm{KI}$ using CRISPR-Cas nuclease (up to $7.1 \mathrm{~kb}$ ) (Table S4) (Menoret et al., 2015; Nakao et al., 2016; Quadros et al., 2017). dsDNA donors were used to insert a 7.1-kb DNA fragment at the target site in $3.1 \%(3 / 97)$ mouse pups (Table S4) (Nakao et al., 2016). HBR using a dsDNA donor occurs through three distinct pathways: homologous recombination $(H R)$, single-stranded annealing (SSA), and microhomology- 
mediated end joining (MMEJ). Each pathway requires a different DNA donor vector construction strategy (Fig. 2).

HR takes advantage of exogenous dsDNA donors or endogenous homologous chromosomes as repair templates (Yoshimi et al., 2014). The exogenous dsDNA donors of HR could be either supercoiled or linear plasmids with long homology arms (Fig. 2 and Table S4) (Canaj et al., 2019). Compared with SSTR, HR requires much longer homology arms (>400 bp, typically $0.5-1 \mathrm{~kb}$ ) (Fig. 2). In human cells, at least a 400-bp homology arm at each end is required for efficient nuclease-induced $\mathrm{HR}$, and the lengthening of homology arms was found to increase the targeted insertion efficiency at some target sites (Hendel et al., 2014; Chu et al., 2015).

Successful gene $\mathrm{KI}$ or precise deletion has been achieved in mouse, rat, rabbit, pig, and human embryos by injecting supercoiled or linear plasmids into zygotes (Table S4). Linear plasmids showed a higher KI efficiency than the supercoiled plasmids, but also led to a higher random integration of donor plasmids (Menoret et al., 2015). In addition, conditional alleles could also be generated efficiently in mice, rats, and pigs (Table S4) (Lee and Lloyd, 2014). To the best of our knowledge, the 631-bp conditional allele, floxed by LoxP, is the largest allele generated by injecting plasmid donor and CRISPR-Cas nuclease (Table S4) (Ma et al., 2014). In theory, a larger conditional allele (up to $7.1 \mathrm{~kb}$ ) could be generated, however, the upper limit (defined as n-bp) of the conditional allele, which could be generated using a plasmid donor, remains to be determined.

Although HR allows for the precise integration of large DNA fragments, the efficiency of HR varies among cell types and species. To increase the efficiency of KI, Yang et al. and Zhang et al. developed new KI strategies, which might occur via the SSA pathway (Yao et al., 2017; Zhang et al., 2017). In this strategy, supercoiled plasmids harboring synthetic gRNA target sites and two 800-bp homology arms were utilized as DNA donors (Fig. 2) (Yao et al., 2017; Zhang et al., 2017). The supercoiled plasmids were linearized in vivo and acted as repair templates (named homology-mediated end joining (HMEJ) strategy, Fig. 2) (Yao et al., 2017; Zhang et al., 2017).

SSA is very similar to MMEJ. First, DSBs and the dsDNA donor are processed via $5^{\prime}$-end resection to reveal long homology arms (>200 bp) (Liskay et al., 1987), which anneal to each other via base pairing (Fig. 1). Then, the gaps between the DSBs and dsDNA donor are filled and the breaks are sealed, leading to the insertion of exogenous DNA (Fig. 1). The enzyme involved in gap filling and break sealing has yet to be elucidated (Yeh et al., 2019).

A later study found that these supercoiled plasmids could be replaced with linear dsDNA with two 800-bp homology arms (named targeted integration with linearized dsDNA (Tild) strategy) (Fig. 2) (Yao et al., 2018b). Both types of DNA donors were capable of efficiently and precisely integrating exogenous DNA at the target site (Fig. 2) (Yao et al., 2018b). The HMEJ and Tild strategy displayed efficient HBR in mouse, monkey, and human embryos (Table S4) (Yao et al., 2017; Yao et al., 2018a, 2018b). However, whether it is possible to generate conditional allele using HMEJ and Tild strategy has not yet been studied, although it is feasible in principle (Table S4) (Yao et al., 2017, 2018a, 2018b).

The construction of plasmid donors with long homology arms is challenging and time-consuming, especially when the target region contains a high GC content or repetitive sequences. In addition, because of the long homology arm, it is difficult to apply PCR and sequencing strategies to screen out the edited cells or animals. A laborious Southern blot assay should be performed to identify and confirm the edited cells or animals.

Precise integration into the target chromosome (PITCh) strategy uses supercoiled plasmids harboring synthetic gRNA target sites and two microhomology arms as the repair template (Nakade et al., 2014). Constructing a PITCh plasmid donor is much more convenient than the HR and SSA plasmid donors because of its short homology arm (5-40 bp) (Nakade et al., 2014). After delivering the supercoiled plasmids and CRISPR-Cas nuclease into the cells, both the supercoiled plasmids and the target site are cleaved. The microhomology arms then aid the integration of the DNA, flanked by the two microhomology arms, through the MMEJ pathway (Fig. 2). First, the DSB and the dsDNA donor are processed by $5^{\prime}$-end resection to reveal a short homology arm, resulting in annealing via base pairing (Fig. 1). Then, DNA polymerase $\theta$ binds the annealed products and fills the gaps between the DSB and dsDNA donor by templated synthesis (Fig. 1). Finally, the DNA breaks are sealed by DNA ligase I or DNA ligase III, resulting in the targeted insertion of exogenous DNA (Fig. 1) (Yeh et al., 2019).

Unlike $H R$, which is active during the late $S / G_{2}$ phases, MMEJ is active during the $G_{1}$ /early $S$ phases. Successful targeted integration by PITCh has been reported in silkworm embryos and zebrafish embryos, whose HR efficiency is low (Nakade et al., 2014). Using the PITCh strategy, a 5-kb DNA fragment was successfully inserted at the target site in $12 \%$ (3/25) mouse zygotes (Table S4) (Aida et al., 2016). The ectopic expression of some MMEJ-related genes (e.g., EXO1, LIG3, PARP1, NBS1, FEN1, BLM, and MRE11A) enhanced MMEJ efficiency in human cells, whereas the ectopic expression of HR-related genes (e.g., RAD51) and SSA-related genes (e.g., RAD52) suppressed MMEJ (Aida et al., 2016). Exonuclease 1 (EXO1) is a $5^{\prime}-3^{\prime}$ exonuclease involved in the end resection of DSB. Co-injected with EXO1 nuclease, the KI efficiency of PITCh increased about 3-fold in mouse zygotes (Table S4) (Aida et al., 2015, 2016; Hisano et al., 2015). Furthermore, a conditional knockout mouse model was generated efficiently (33.3\%) via the PITCh strategy (Table S4) (Aida et al., 2016). Therefore, the PITCh strategy allows for both the efficient generation and convenient identification of large fragment $\mathrm{KI}$ animal models. 
Although HBR efficiency varied site by site, the above four strategies (HR, HMEJ, Tild, and PITCh) showed different HBR efficiencies in mammalian embryos. Compared with conventional HR using supercoiled plasmid, Tild achieved a 6.4-fold higher HBR efficiency in human embryos (Yao et al., 2018b). Comparing the HBR efficiency of PITCh and HMEJ revealed that HMEJ is much more efficient (1.9-3.7-fold) than PITCh at three target sites (Actb, Dbh, and Sox2) in mouse embryos (Table S4) (Yao et al., 2017). Moreover, Tild (targeted integration with linearized dsDNA) showed a higher (1.6-3.3-fold) HBR efficiency than HMEJ (homologymediated end joining) at three sites ( $C d x 2, A c t b$, and Sp8) in mouse embryos (Table S4) (Yao et al., 2018b). Thus, the HBR efficiency in mammalian embryos may follow the order: Tild $>$ HMEJ $>$ PITCh $\geq$ HR. Based on current data, the Tild and HMEJ strategies may be the first choice for large DNA fragment knock-in in large mammalian animal and human embryos.

\section{ENHANCED HOMOLOGY-BASED REPAIR THROUGH TETHERING OF DNA DONOR}

As $\mathrm{HBR}$ requires the presence of a DNA donor at the target site, many efforts have been devoted to increasing the concentration of DNA donors at target sites to enhance HBR. Recently, several methods have been developed to recruit DNA donors to target sites by tethering DNA donors to the gRNA-Cas9 complex (Fig. 4).

The Cas9-avidin biotin ssDNA (CAB) system takes advantage of Cas9-Avidin fusion protein and ssDNA labeled with biotin at the $5^{\prime}$ end (Fig. 4A) (Ma et al., 2017b). The interaction between avidin and biotin recruits a DNA donor to the target site, resulting in an increased HBR efficiency (2-5fold) in human cells (Ma et al., 2017b). Furthermore, the CAB system enhanced HBR efficiency in mouse embryos by 3-fold compared with conventional CRISPR-Cas9 nuclease (Ma et al., 2017b). A similar system, based on the interaction between monomeric streptavidin (mSA) and biotin, was developed. Unlike the CAB system, the Cas9-mSA BIOPCR-donor CRISPR system utilized biotin-labeled dsDNA donor as a repair template (Fig. 4B) (Gu et al., 2018; Roche et al., 2018). The biotin-labeled dsDNA donor was generated by PCR amplification using primers with 5'-biotin modification. The Cas9-mSA BIO-PCR-donor CRISPR system enhanced the HBR efficiency in human cells and in 2-cell mouse embryos (1.6-3.8-fold) (Gu et al., 2018; Roche et al., 2018).

In addition to avidin-biotin non-covalent interactions, other protein-substrate covalent interactions have been used to recruit DNA donors. The ribonucleoprotein DNA (RNPD) system is composed of gRNA, Cas9-SNAP fusion protein, and $\mathrm{O}^{6}$-benzylguanine (BG)-labeled ssODN (Savic et al., 2018). SNAP peptide could bind the $O^{6}$-benzylguanine (BG)-labeled ssODN covalently, recruiting ssODN to the target site. The RNPD system enhanced the HBR efficiency in human HEK-293T cells (7-24-fold), K562 cells (17-fold), and mouse embryonic stem cells (mESCs) (2-6-fold) (Savic et al., 2018). Intriguingly, an increase in the ssODN concentration in the nucleus was sufficient to enhance HBR efficiency (Savic et al., 2018). Similarly, the Cas9-PCV system is based on the covalent interaction between the porcine circovirus 2 (PCV) Rep protein and its recognition DNA sequence (Aird et al., 2018). ssODN with a PCV recognition sequence is recruited to the target site by the gRNA-Cas9PCV complex, resulting in enhanced HBR efficiency (Aird et al., 2018).

Recently, a method based on noncanonical amino acid (ncAA) modified Cas9 was developed (Ling et al., 2020). In this Cas9-AeF DBCO-adaptor ssODN system, the G1367 residue of Cas9 was replaced with a ncAA named 4-(2-azidoethoxy)-I-phenylalanine (AeF). Purified Cas9-AeF proteins were then incubated with gRNA, DBCO-modified ssDNA adaptor, and a ssODN donor. The azide group of AeF reacts with the alkyne of dibenzylcyclooctyne (DBCO), linking DBCO-modified ssDNA adaptors to Cas9. The linked ssDNA adaptor then recruits the ssODN donor by base pairing, tethering sSODN to the gRNA-Cas9 complex. Transfecting the Cas9-AeF DBCO-adaptor ssODN system into human cells led to a 10-fold increase in HBR efficiency (Ling et al., 2020). Moreover, injecting the Cas9-AeF DBCO-adaptor ssODN system into mouse embryos also led to a 2.3-fold increase in HBR efficiency (Ling et al., 2020).

Like Cas9 protein, gRNA can also be used to recruit DNA donors. The S1m aptamer, an RNA fragment capable of binding streptavidin tetramer, is installed in the first stem loop of the gRNA. Biotin-labeled ssDNA donor interacts with streptavidin tetramer, which is recruited to the gRNA-Cas9 complex by the S1m aptamer (Fig. 4F) (Carlson-Stevermer et al., 2017). Delivering this S1mplex system into human cells led to an enhanced HBR/indel frequency ratio (2.718.4-fold); however, this led to decreased absolute HBR efficiency (Carlson-Stevermer et al., 2017). In addition, donor DNA can be directly conjugated with guide RNA. In the Cas9 gRNA-donor DNA conjugate system, donor DNA is conjugated with the 5' end of crRNA in the crRNA-tracrRNA dimer, forming a gRNA-donor DNA conjugate (gDonor) (Lee et al., 2017). Purified Cas9 protein is able to assemble with gDonor in vitro (Lee et al., 2017) (Fig. 4G). After delivering this assembled complex into human cells, a obvious increase in HBR efficiency was observed (Lee et al., 2017).

Of the seven systems mentioned above, only three systems (CAB system, Cas9-mSA BIO-PCR-donor CRISPR system, and Cas9-AeF DBCO-adaptor ssODN system) have been proven to be effective in mouse embryos. It would be interesting to compare the efficiency of all seven systems in mammalian embryos under the same circumstances. These results will help us to determine the most efficient and safe system for mammalian embryo genome editing. 
A

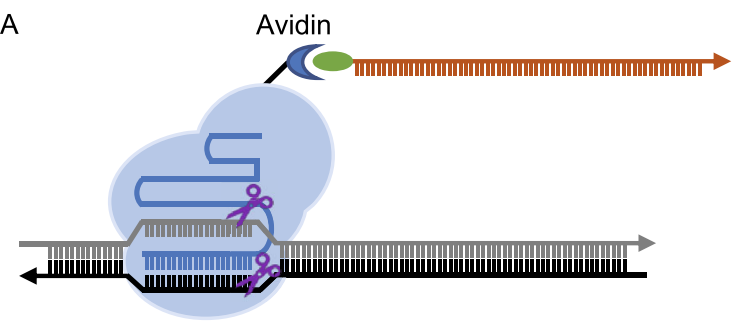

Cas9-avidin biotin ssDNA system (CAB)

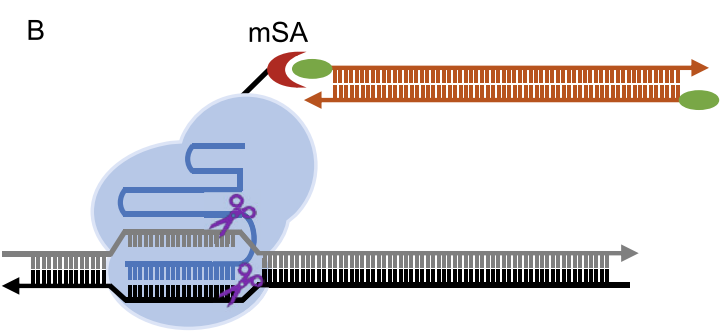

Cas9-mSA BIO-PCR-donor CRISPR system
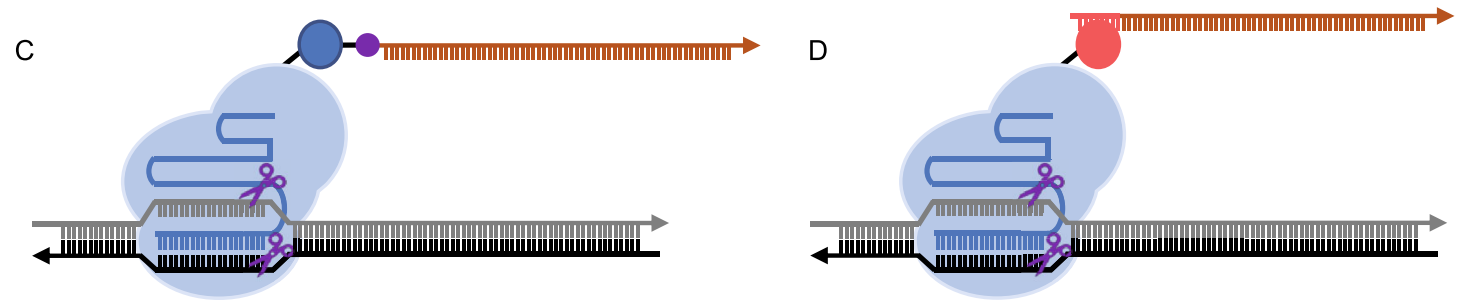

RNPD system

Cas9-PCV system

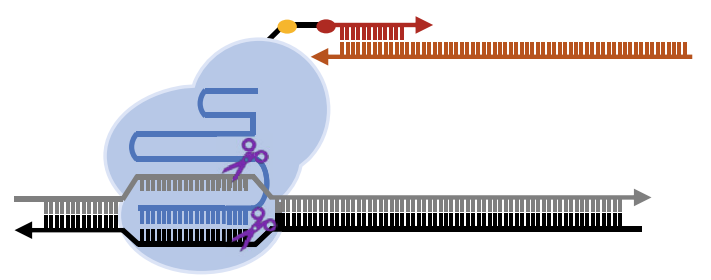

Cas9-AeF DBCO-adaptor ssODN system

F

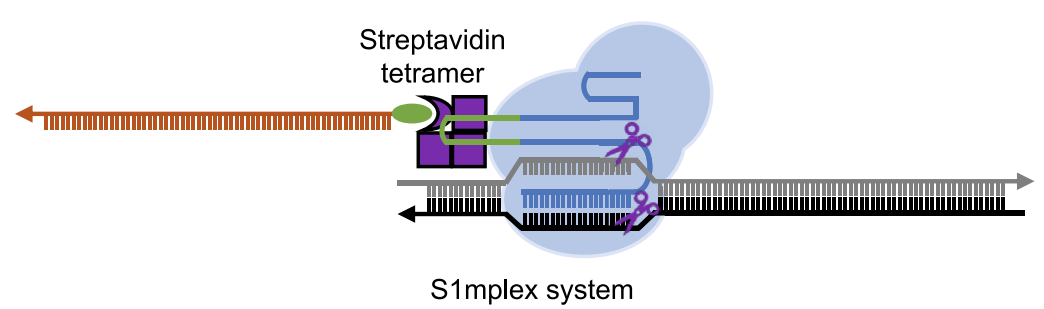

G

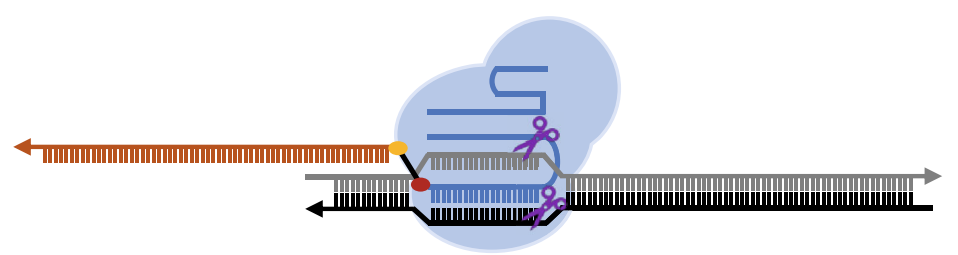

Cas9 gRNA-donor DNA conjugate system (Cas9-gDonor)

Figure 4. Different methods to recruit donor DNA to target site. (A) Cas9-avidin biotin ssDNA (CAB) system. Biotin, green oval. (B) Cas9-mSA BIO-PCR-donor CRISPR system. mSA, monomeric streptavidin. (C) The ribonucleoprotein DNA (RNPD) system. SNAP peptide, blue circle. $0^{6}$-benzylguanine (BG), purple circle. Covalent bond between SNAP peptide and $\mathrm{O}^{6}$-benzylguanine (BG), black line. (D) The Cas9-PCV system. PCV, porcine circovirus 2 Rep protein, is shown with pink circle. PCV recognition sequence, pink line. (E) Cas9-AeF DBCO-adaptor ssODN system. AeF, noncanonical amino acid 4-(2-azidoethoxy)-l-phenylalanine. Azide group of AeF, golden oval. DBCO, red oval. Adaptor, red line. (F) S1mplex system. Chimeric RNA, composed of gRNA and S1m aptamer, recruits biotin-labeled ssDNA donor to the target site by interacting with the streptavidin tetramer (purple box). S1m aptamer, green line. (G) Guide RNA donor DNA conjugate (gDonor) system. Azide group, golden oval. DBCO, red oval. gRNA, blue line. DNA donor, orange line. The direction of the arrow represents the $5^{\prime}$ to $3^{\prime}$ direction. 


\section{HOMOLOGY-BASED REPAIR USING ENDOGENOUS HOMOLOGOUS DNA}

In addition to exogenous DNA donors, endogenous homologous DNA can be utilized as an HBR template in mouse and human tripronuclear zygotes (Wu et al., 2013; Liang et al., 2015). Our group found that human tripronuclear zygotes prefer endogenous homologous DNA (HBD gene) to ssODN donors while editing $H B B$ gene in human tripronuclear zygotes (Liang et al., 2015). The HBD gene used as a repair template may be from either the paternal or maternal chromosome (Liang et al., 2015). Later, Mitalipov et al. found that human diploid zygotes prefer to use the wild-type maternal chromosome than ssODN as repair templates (Ma et al., 2017a). However, owing to the rapid cleavage of target DNA by CRISPR-Cas nuclease and the spatial distance between paternal and maternal chromosomes in one-cell embryos, whether the maternal chromosome could be utilized as repair templates remains a topic of controversy (Ma et al., 2017a, 2018; Adikusuma et al., 2018; Egli et al., 2018; Reichmann et al., 2018). An alternative interpretation of Mitalipov's data is that the paternal allele failed to be amplified due to the loss of segmental paternal chromosome as a result of large DNA fragment deletions, segmental paternal chromosome gain due to DNA fragment duplication, complete paternal chromosome loss, or translocations (Adikusuma et al., 2018; Egli et al., 2018; Alanis-Lobato et al., 2020; Zuccaro et al., 2020). Recently, Egli et al. found that templated repair using maternal chromosomes may occur in two-cell human embryos when the paternal and maternal genomes are in the same nucleus. However, its efficiency is relatively low ( 7\%) (Zuccaro et al., 2020). At the one-cell stage, templated repair using maternal chromosome did not occur due to separation of the male and female pronucleus (Zuccaro et al., 2020). Moreover, they found that even a single CRISPR-Cas nuclease-induced cut could lead to frequent loss of targeted chromosomes by destabilizing the entire chromosome in human early embryos, resulting in the failure of targeted DNA amplification using PCR (Zuccaro et al., 2020). It is noteworthy that these findings also underscore the risk of large fragment deletion and aneuploidy while editing the human embryo genome using CRISPR-Cas nuclease (Zuccaro et al., 2020). Further mechanistic and methodological studies are needed to develop techniques to eliminate large fragment deletions and aneuploidy while editing the human embryo genome using CRISPR-Cas nuclease.

HBR using endogenous homologous DNA template can reduce the efficiency of generating intended genome modifications using exogenous DNA donors. However, it may also allow to correct gene mutations through templated repair using endogenous homologous DNA template (Liang et al., 2015). Thus, depending on the purpose of genome editing, HBR using endogenous homologous sequences could be either beneficial or harmful.

\section{RNA-TEMPLATED DNA REPAIR: PRIME EDITING}

In addition to DNA, RNA can be utilized as repair templates. Recently, Liu et al. developed prime editors, consisting of prime editor (PE) protein and prime editing guide RNA (pegRNA), to edit the genome without inducing DNA DSB (Anzalone et al., 2019). PE protein is composed of Cas9 nickase (Cas9n-H840A) and M-MLV reverse transcriptase variant (M-MLV D200N + L603W + T330P + T306K + W313F). pegRNA is comprised of four parts: a guide sequence, a gRNA backbone, a reverse transcription (RT) template, and a primer binding site (PBS). Under the guidance of pegRNA, the PE:pegRNA complex binds the target strand at the target site, resulting in the displacement of the non-target strand. Then, the Cas9n-H840A RuvC nuclease domain of the PE protein cleaves the non-target strand, exposing a 3'-hydorxyl group on the non-target strand. The cleaved and displaced non-target strand will hybridize with the PBS of pegRNA by base pairing, and the 3'-hydroxyl group on this strand will be used to prime reverse transcription by the MMLV reverse transcriptase domain. Thus, the edit encoded in the RT template of the pegRNA will be copied into the target site. The newly synthesized DNA will displace the wild-type sequence at the target site, resulting in a 5'-flap, which is cleaved and sealed by the DNA repair enzyme.

Based on this strategy, prime editors have been used to generate precise point mutations, small deletions ( $\leq 80 \mathrm{bp}$ ), and insertions ( $\leq 40 \mathrm{bp}$ ) in HEK-293T cells (Anzalone et al., 2019). However, for unknown reasons, the efficiency of the prime editor is very low in human iPSCs and mouse embryos (Liu et al., 2020; Surun et al., 2020). Therefore, further study is needed to improve the efficiency of prime editors in mammalian embryos.

\section{CONCLUSION AND PERSPECTIVES}

ssDNA and dsDNA donors have both advantages and disadvantages. For example, ssDNA may introduce sequence errors in homology arms (sSODN and IssDNA), LoxP sites (ssODN and IssDNA), and the LoxP floxed region (ssODN and IssDNA) (Lanza et al., 2018). ssDNA could be used to generate $\mathrm{KI}$ mice by electroporation-based delivery (e.g., zygote electroporation and iGONAD), whereas successful genome editing using dsDNA donor delivered by zygote electroporation has yet to be reported. To date, dsDNA donors have been delivered into zygotes by microinjection (Remy et al., 2017; Miyasaka et al., 2018; Ohtsuka et al., 2018). Therefore, delivering ssDNA donors into zygotes is much more convenient than delivering dsDNA donors. In addition, dsDNA donors often lead to a higher cell toxicity and more off-target integration (Chen et al., 2011). To provide a guideline for the design of HBR DNA donors for mammalian embryo genome editing, the DNA donor designs and editing outcomes have been summarized in Table 1. 
Table 1. Summary of donor DNA design and editing outcomes in mammalian embryos.

\begin{tabular}{|c|c|c|c|c|c|}
\hline \multirow[t]{2}{*}{ Donor DNA } & \multirow[t]{2}{*}{ 5' homology arm } & \multirow[t]{2}{*}{ 3' homology arm } & \multicolumn{3}{|c|}{ Major editing type } \\
\hline & & & Point mutation & Insertion & Conditional allele \\
\hline ssODN & $\geq 30 \mathrm{nt}$ & $\geq 30 \mathrm{nt}$ & $\mathrm{OK}$ & $\leq 100 \mathrm{nt}$ & Not efficient \\
\hline IssDNA & $\geq 55 \mathrm{nt}$ & $\geq 55 \mathrm{nt}$ & OK & $\leq 1,368 \mathrm{nt}$ & OK \\
\hline AAV & $\geq 475 \mathrm{nt}$ & $\geq 475 \mathrm{nt}$ & OK & $\leq 3,300 \mathrm{nt}$ & Unknown \\
\hline Supercoiled plasmid ${ }^{a}$ & $\geq 500 \mathrm{bp}$ & $\geq 500 \mathrm{bp}$ & Unnecessary & $\leq 7,100 \mathrm{bp}$ & OK \\
\hline Supercoiled plasmid ${ }^{\mathrm{b}}$ & 800 bp & $800 \mathrm{bp}$ & Unnecessary & $\sim 800 \mathrm{bp}$ & Unknown \\
\hline Supercoiled plasmid ${ }^{\mathrm{C}}$ & $40 \mathrm{bp}$ & $40 \mathrm{bp}$ & Unnecessary & $\leq 5,000 \mathrm{bp}$ & OK \\
\hline Linear plasmid $^{a}$ & $\geq 500 \mathrm{bp}$ & $\geq 500 \mathrm{bp}$ & Unnecessary & $\leq 7,100 \mathrm{bp}$ & OK \\
\hline Linear dsDNA $^{d}$ & 800 bp & 800 bp & Unnecessary & $\leq 6,000$ bp & Unknown \\
\hline
\end{tabular}

Note: It is unnecessary to used plasmids and linear dsDNA to generate point mutation mammals. a, HR. b, HMEJ. c, PITCh. d, Tild.

Furthermore, a decision tree illustrating the main considerations for DNA donor design is provided in Fig. 5.

Recently, lyer et al. produced long circular single-stranded DNA (cssDNA) by superinfecting $E$. coli cells containing phagemids with VCSM13 helper phages (lyer et al., 2019). The DNA replication machinery provided by the helper phage recognizes the $\mathrm{f1}$ origin of the phagemids, producing phages with circular single-stranded DNA. These phages are then harvested to purify the cssDNA. Long cssDNA donors have been used as HBR templates in human HEK293T cells and K562 cells. Compared with the linear ssDNA donor, long cssDNA results in a much higher HBR efficiency (lyer et al., 2019). Exploring whether long cssDNA also enhance HBR in mammalian embryos could provide new avenues for mammalian embryo genome editing.

In addition to the DNA donor, the cutting efficiency of CRISPR-Cas nuclease is a major factor that affects HBR efficiency (Lanza et al., 2018). Therefore, before designing DNA donors, highly active gRNAs should be screened by delivering them together with CRISPR-Cas nuclease into cells or embryos. In addition, changing the formulation of CRISPR-Cas nuclease could also increase its cutting efficiency. The ctRNP (crRNA + tracrRNA + Cas9 protein) complex could result in a higher $\mathrm{KI}$ efficiency than the Cas9mRNA/gRNA mixture and gRNP (gRNA + Cas9 protein) complex (Quadros et al., 2017).

Additionally, controlling the time of CRISPR-Cas nuclease and DNA donor delivery also helps to increase HBR efficiency and reduce mosaicism (Ma et al., 2017a; Gu et al., 2018). It has been reported that microinjection at the two-cell stage increased the $\mathrm{KI}$ efficiency over 10-fold compared to that of the one-cell stage in mice (Gu et al., 2018). However, whether injecting CRISPR-Cas nuclease and DNA donor into the $S / G_{2}$ phase (PN3-PN5) zygotes results in a higher editing efficiency than $\mathrm{G} 1$ phase (PNO-PN2) zygotes remains to be determined (Wossidlo et al., 2011).

In addition, the specificity of gRNA is also an important consideration. Cas9 nickase (Cas9n), which only cleaves one strand of the DNA double helix, has an increased specificity but is not routinely used for $\mathrm{KI}$ because of its low efficiency (Lee and Lloyd, 2014; Cornu et al., 2017; Kan et al., 2017). It is worth noting that both ssDNA and dsDNA donors are able to integrate randomly into the genome, particularly at off-target sites (Quadros et al., 2017; Lanza et al., 2018; Li et al., 2019). Therefore, it is important to check the random integration events of DNA donors in F1generation animals.

In addition to CRISPR-Cas nuclease-mediated HBR, base editors have been used to efficiently generate point mutations without inducing DNA DSB in mammalian and human embryos (Kim et al., 2017b; Liang et al., 2017, 2018; Liu et al., 2018b, 2018c; Ryu et al., 2018; Yang et al., 2018; Anzalone et al., 2020). Cytidine base editor (CBE), which is composed of cytidine deaminase, Cas9n-D10A, and a uracil DNA glycosylase inhibitor (UGI) fusion protein, is able to catalyze C-to-T conversion in the activity window under the guidance of gRNA (Komor et al., 2016), while C-to-G base editor (CGBE), which is composed of cytidine deaminase and Cas9n-D10A fusion protein, can catalyze C-to-G conversion in the activity window (Kurt et al., 2020; Zhao et al., 2020b). Similarly, an adenine base editor (ABE), which is composed of adenine deaminase and Cas9n-D10A fusion protein, catalyzes A-to-G conversion in the activity window (Gaudelli et al., 2017). When editing the genome of mammalian and human embryos using base editors, care must be taken with the off-target DNA and RNA mutations (Kim et al., 2017a, 2017c, 2019; Grunewald et al., 2019a; Jin et al., 2019; Liang and Huang, 2019; Liang et al., 2019a, 2019b; Zhou et al., 2019; Zuo et al., 2019). High-fidelity base editor variants with lower off-target effects should be used (Liang et al., 2017; Grunewald et al., 2019b; Liang and Huang, 2019; Liang et al., 2019a; Rees et al., 2019; Zhou et al., 2019; Doman et al., 2020; Yu et al., 2020; Zuo et al., 2020). A recent review by Anzalone et al. is an excellent guide to choose the appropriate base editors for genome editing (Anzalone et al., 2020). 


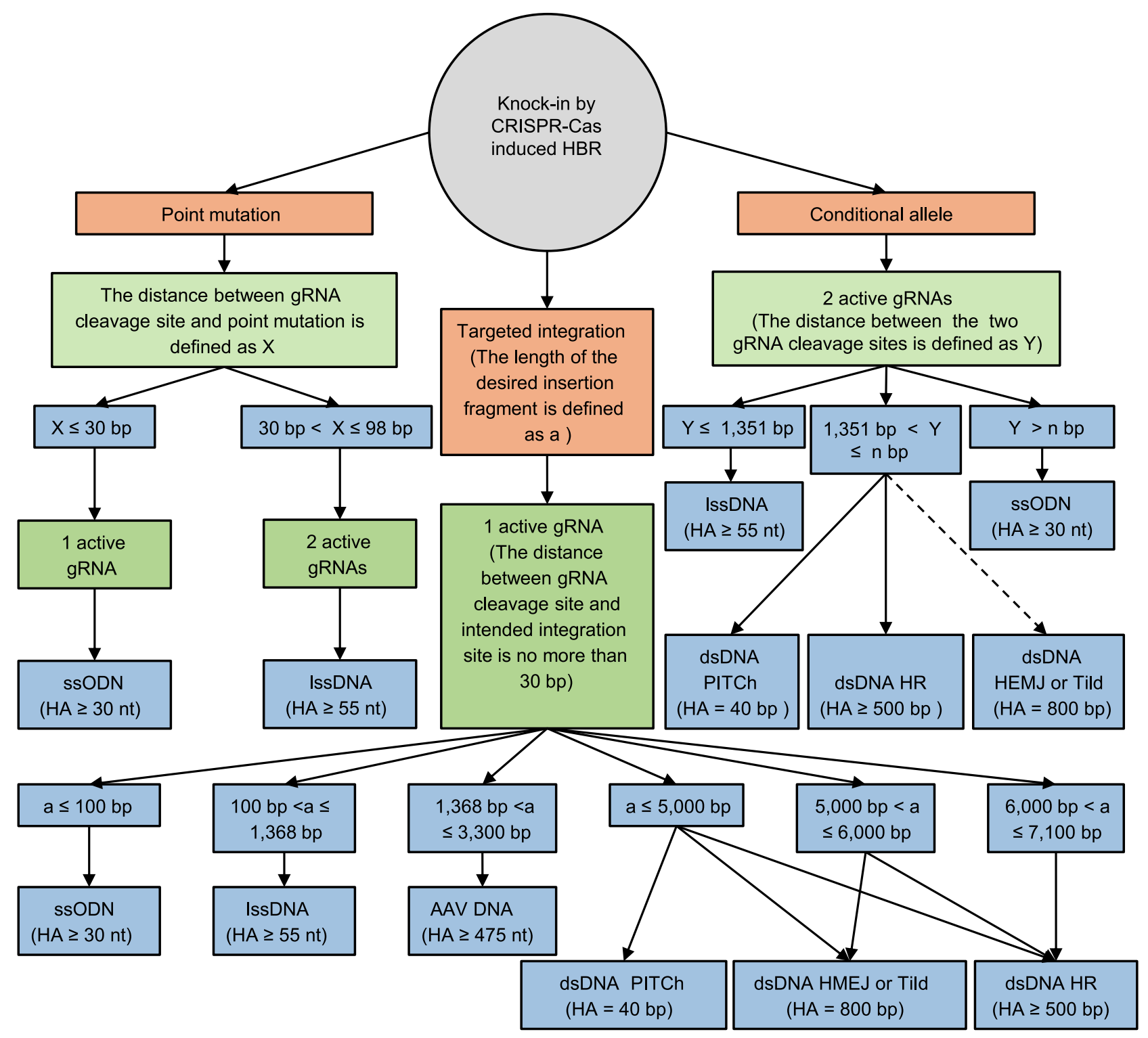

Figure 5. Decision tree for the selection of the HBR donor vector. HBR donor vectors should be designed based on several critical considerations, including the desired knock-in type (point mutation, targeted integration and conditional allele), the distance between gRNA cleavage site and the desired edit site $(X)$, the length of the desired insertion fragment (a), and the distance between the two gRNA cleavage sites $(Y)$. For point mutations, if $X \leq 30 \mathrm{bp}$, a ssODN donor is recommended. However, if $30<\mathrm{X} \leq 98 \mathrm{bp}$, a IssDNA donor is recommended. For targeted integration, if $\mathrm{X} \leq 30 \mathrm{bp}$, strategies that used one active gRNA are recommended. If a $\leq$ $100 \mathrm{bp}$, sSODN is recommended. If the length of the desired insertion fragment increases, other strategies are recommended, as shown in this figure. For targeted integration, if $X>30 \mathrm{bp}$, strategies similar to conditional allele generation should be used. For conditional allele generation, two active gRNAs should be identified at first. If the distance between the two active gRNAs ( $Y$ ) is no more than $1,351 \mathrm{bp}$, IssDNA is recommended. The upper limit of the conditional allele (n bp) that could be generated using dsDNA remains to be investigated. If $1,351<\mathrm{Y} \leq \mathrm{n}$ bp, a dsDNA donor is recommended (e.g., PITCh and HR). Although HEMJ and Tild have not yet been applied for generating conditional allele in mammalian embryos, HEMJ and Tild could be used to generate conditional allele in theory. Although generating conditional alleles using SSODN is inefficient, it could be used to generate very large conditional allele $(\mathrm{Y}>\mathrm{n}$ bp). The solid lines indicate strategies that have been demonstrated in mammalian embryos. The dashed line indicates possible strategies (HEMJ and Tild) that remain to be tested in mammalian embryos. 
Moreover, the targeted insertion of large DNA fragments by CRISPR-associated transposases has been reported in E. coli (Klompe et al., 2019; Strecker et al., 2019). However, whether these CRISPR-associated transposases can lead to targeted insertion in eukaryotic cells has yet to be elucidated. Thus, an in-depth study of CRISPR-associated transposases may enable the targeted insertion of large DNA fragments in eukaryotic cells and mammalian embryos.

The advent of genome editing tools has enabled the generation of genome-edited mammalian animals, which will not only help to improve our understanding of fundamental biology underlying life and disease, but also promote advances in livestock farming. These tools can be used for the repair disease mutations in situ, with the potential to make incurable diseases curable and promote the development of precision medicine in the future.

\section{ABBREVIATIONS}

AAV, adeno-associated virus; c-NHEJ, classical non-homologous end joining; DSB, double-stranded break; dsDNA, double-stranded DNA; HBR, homology-based repair; HITI, homology-independent targeted integration; HMEJ, homology-mediated end joining; HR, homologous recombination; HSPCs, human hematopoietic stem and progenitor cells; iVTRT, in vitro transcription and reverse transcription; KI, knock-in; LNA, locked nucleic acid; IssDNA, long single-stranded DNA; MMEJ, microhomology-mediated end joining; PITCh, precise integration into the target chromosome; PS, phosphorothioate; SDSA, synthesis-dependent strand annealing; SSA, single-stranded annealing; ssODN, single-stranded oligodeoxynucleotide; SSTR, single-stranded templated repair; Tild, targeted integration with linearized dsDNA; cssDNA, circular single-stranded DNA; Cas9n, Cas9 nickase; CBE, cytidine base editor; UGI, uracil DNA glycosylase inhibitor; CGBE, C-to-G base editor; $A B E$, adenine base editor.

\section{DECLARATIONS}

\section{Funding}

This work was supported by the Key Technologies Research and Development Program (2017YFC1001901), the Guangdong Special Support Program (2019BT02Y276), Guangdong Basic and Applied Basic Research Foundation (2018A030313370 and 2021A1515010759), the National Natural Science Foundation of China (31971365), and the Guangzhou Science and Technology Project (201803010020).

\section{Conflicts of interest}

The authors declare no competing interests.

\section{AUTHORS' CONTRIBUTIONS}

X Z., T. L., J. O., J. H., and P. L. discussed the structure and organization of the manuscript. X. Z., J. H., and P. L. wrote the manuscript. All authors commented on the manuscript.

\section{OPEN ACCESS}

This article is licensed under a Creative Commons Attribution 4.0 International License, which permits use, sharing, adaptation, distribution and reproduction in any medium or format, as long as you give appropriate credit to the original author(s) and the source, provide a link to the Creative Commons licence, and indicate if changes were made. The images or other third party material in this article are included in the article's Creative Commons licence, unless indicated otherwise in a credit line to the material. If material is not included in the article's Creative Commons licence and your intended use is not permitted by statutory regulation or exceeds the permitted use, you will need to obtain permission directly from the copyright holder. To view a copy of this licence, visit http:// creativecommons.org/licenses/by/4.0/.

\section{REFERENCES}

Adikusuma F, Piltz S, Corbett MA, Turvey M, McColl SR, Helbig KJ, Beard MR, Hughes J, Pomerantz RT, Thomas PQ (2018) Large deletions induced by Cas9 cleavage. Nature 560:E8-E9

Aida T, Chiyo K, Usami T, Ishikubo H, Imahashi R, Wada Y, Tanaka KF, Sakuma T, Yamamoto T, Tanaka K (2015) Cloning-free CRISPR/Cas system facilitates functional cassette knock-in in mice. Genome Biol 16:87

Aida T, Nakade S, Sakuma T, Izu Y, Oishi A, Mochida K, Ishikubo H, Usami T, Aizawa H, Yamamoto T et al (2016) Gene cassette knock-in in mammalian cells and zygotes by enhanced MMEJ. BMC Genomics 17:979

Aird EJ, Lovendahl KN, St Martin A, Harris RS, Gordon WR (2018) Increasing Cas9-mediated homology-directed repair efficiency through covalent tethering of DNA repair template. Commun Biol $1: 54$

Alanis-Lobato G, Zohren J, Mccarthy A, Fogarty NME, Kubikova N, Hardman E, Greco M, Wells D, Turner JMA, Niakan KK (2020) Frequent loss-of-heterozygosity in CRISPR-Cas9-edited early human embryos. bioRxiv.

Anzalone AV, Randolph PB, Davis JR, Sousa AA, Koblan LW, Levy JM, Chen PJ, Wilson C, Newby GA, Raguram A et al (2019) Search-and-replace genome editing without double-strand breaks or donor DNA. Nature 576(7785):149-157

Anzalone AV, Koblan LW, Liu DR (2020) Genome editing with CRISPR-Cas nucleases, base editors, transposases and prime editors. Nat Biotechnol 38:824-844

Bak RO, Porteus MH (2017) CRISPR-Mediated Integration of Large Gene Cassettes Using AAV Donor Vectors. Cell Rep 20:750-756

Baltimore D, Berg P, Botchan M, Carroll D, Charo RA, Church G, Corn JE, Daley GQ, Doudna JA, Fenner M et al (2015) Biotechnology. A prudent path forward for genomic engineering and germline gene modification. Science 348:36-38 
Bedell VM, Wang Y, Campbell JM, Poshusta TL, Starker CG, Krug RG 2nd, Tan W, Penheiter SG, Ma AC, Leung AY et al (2012) In vivo genome editing using a high-efficiency TALEN system. Nature 491:114-118

Bennardo N, Cheng A, Huang N, Stark JM (2008) Alternative-NHEJ is a mechanistically distinct pathway of mammalian chromosome break repair. PLoS Genet 4:

Bothmer A, Phadke T, Barrera LA, Margulies CM, Lee CS, Buquicchio F, Moss S, Abdulkerim HS, Selleck W, Jayaram H et al (2017) Characterization of the interplay between DNA repair and CRISPR/Cas9-induced DNA lesions at an endogenous locus. Nat Commun 8:13905

Canaj H, Hussmann JA, Li H, Beckman KA, Goodrich L, Cho NH, Li YJ, Santos DA, McGeever A, Stewart EM et al (2019) Deep profiling reveals substantial heterogeneity of integration outcomes in CRISPR knock-in experiments. BioRxiv.

Carlson-Stevermer J, Abdeen AA, Kohlenberg L, Goedland M, Molugu K, Lou M, Saha K (2017) Assembly of CRISPR ribonucleoproteins with biotinylated oligonucleotides via an RNA aptamer for precise gene editing. Nat Commun 8:1711

Ceccaldi R, Rondinelli B, D'Andrea AD (2016) Repair pathway choices and consequences at the double-strand break. Trends Cell Biol 26:52-64

Chang HHY, Pannunzio NR, Adachi N, Lieber MR (2017) Nonhomologous DNA end joining and alternative pathways to double-strand break repair. Nat Rev Mol Cell Biol 18:495-506

Chen F, Pruett-Miller SM, Huang Y, Gjoka M, Duda K, Taunton J, Collingwood TN, Frodin M, Davis GD (2011) High-frequency genome editing using ssDNA oligonucleotides with zinc-finger nucleases. Nat Methods 8:753-755

Chen JS, Dagdas YS, Kleinstiver BP, Welch MM, Sousa AA, Harrington LB, Sternberg SH, Joung JK, Yildiz A, Doudna JA (2017) Enhanced proofreading governs CRISPR-Cas9 targeting accuracy. Nature 550:407-410

Chen S, Sun S, Moonen D, Lee C, Lee AY, Schaffer DV, He L (2019) CRISPR-READI: Efficient Generation of Knockin Mice by CRISPR RNP Electroporation and AAV Donor Infection. Cell Rep 27(3780-3789):

Chen Y, Zhi S, Liu W, Wen J, Hu S, Cao T, Sun H, Li Y, Huang L, Liu $Y$ et al (2020) Development of highly efficient dual-AAV split adenosine base editor for in vivo gene therapy. Small Methods 4 (9):2000309

Choulika A, Perrin A, Dujon B, Nicolas JF (1995) Induction of homologous recombination in mammalian chromosomes by using the I-Scel system of Saccharomyces cerevisiae. Mol Cell Biol 15:1968-1973

Chu VT, Weber T, Wefers B, Wurst W, Sander S, Rajewsky K, Kuhn $R$ (2015) Increasing the efficiency of homology-directed repair for CRISPR-Cas9-induced precise gene editing in mammalian cells. Nat Biotechnol 33:543-548

Codner GF, Mianne J, Caulder A, Loeffler J, Fell R, King R, Allan AJ, Mackenzie M, Pike FJ, McCabe CV et al (2018) Application of long single-stranded DNA donors in genome editing: generation and validation of mouse mutants. BMC Biol 16:70

Cong L, Ran FA, Cox D, Lin S, Barretto R, Habib N, Hsu PD, Wu X, Jiang W, Marraffini LA et al (2013) Multiplex genome engineering using CRISPR/Cas systems. Science 339:819-823
Cornu TI, Mussolino C, Cathomen T (2017) Refining strategies to translate genome editing to the clinic. Nat Med 23:415-423

De Ravin SS, Li L, Wu X, Choi U, Allen C, Koontz S, Lee J, Theobald-Whiting N, Chu J, Garofalo M et al (2017) CRISPRCas9 gene repair of hematopoietic stem cells from patients with X-linked chronic granulomatous disease. Sci Transl Med 9: eaah3480

Dever DP, Bak RO, Reinisch A, Camarena J, Washington G, Nicolas CE, Pavel-Dinu M, Saxena N, Wilkens AB, Mantri S et al (2016) CRISPR/Cas9 beta-globin gene targeting in human haematopoietic stem cells. Nature 539:384-389

DeWitt MA, Magis W, Bray NL, Wang T, Berman JR, Urbinati F, Heo SJ, Mitros T, Munoz DP, Boffelli D et al (2016) Selection-free genome editing of the sickle mutation in human adult hematopoietic stem/progenitor cells. Sci Transl Med 8:

Doman JL, Raguram A, Newby GA, Liu DR (2020) Evaluation and minimization of Cas9-independent off-target DNA editing by cytosine base editors. Nat Biotechnol 38:620-628

Doudna JA (2020) The promise and challenge of therapeutic genome editing. Nature 578:229-236

Egli D, Zuccaro MV, Kosicki M, Church GM, Bradley A, Jasin M (2018) Inter-homologue repair in fertilized human eggs? Nature 560:E5-E7

Gaj T, Gersbach CA, Barbas CF 3rd (2013) ZFN, TALEN, and CRISPR/Cas-based methods for genome engineering. Trends Biotechnol 31:397-405

Gaj T, Staahl BT, Rodrigues GMC, Limsirichai P, Ekman FK, Doudna JA, Schaffer DV (2017) Targeted gene knock-in by homologydirected genome editing using Cas9 ribonucleoprotein and AAV donor delivery. Nucleic Acids Res 45:

Gaudelli NM, Komor AC, Rees HA, Packer MS, Badran AH, Bryson DI, Liu DR (2017) Programmable base editing of $A^{*} T$ to $G^{*} C$ in genomic DNA without DNA cleavage. Nature 551:464-471

Ge XA, Hunter CP (2019) Efficient homologous recombination in mice using long single stranded DNA and CRISPR Cas9 nickase. G3 (Bethesda) 9:281-286

Grunewald J, Zhou R, Garcia SP, Iyer S, Lareau CA, Aryee MJ, Joung JK (2019a) Transcriptome-wide off-target RNA editing induced by CRISPR-guided DNA base editors. Nature 569:433437

Grunewald J, Zhou R, Iyer S, Lareau CA, Garcia SP, Aryee MJ, Joung JK (2019b) CRISPR DNA base editors with reduced RNA off-target and self-editing activities. Nat Biotechnol 37:1041-1048

Gu B, Posfai E, Rossant J (2018) Efficient generation of targeted large insertions by microinjection into two-cell-stage mouse embryos. Nat Biotechnol 36:632-637

Gurumurthy CB, O'Brien AR, Quadros RM, Adams J Jr, Alcaide P, Ayabe S, Ballard J, Batra SK, Beauchamp MC, Becker KA et al (2019) Reproducibility of CRISPR-Cas9 methods for generation of conditional mouse alleles: a multi-center evaluation. Genome Biol 20:171

Hendel A, Kildebeck EJ, Fine EJ, Clark J, Punjya N, Sebastiano V, Bao G, Porteus MH (2014) Quantifying genome-editing outcomes at endogenous loci with SMRT sequencing. Cell Rep 7:293-305

Hisano Y, Sakuma T, Nakade S, Ohga R, Ota S, Okamoto H, Yamamoto T, Kawahara A (2015) Precise in-frame integration of 
exogenous DNA mediated by CRISPR/Cas9 system in zebrafish. Sci Rep 5:8841

lyer S, Mir A, Vega-Badillo J, Roscoe BP, Ibraheim R, Zhu LHJ, Lee JY, Liu PP, Luk K, Mintzer E et al (2019) Efficient homologydirected repair with circular SsDNA donors. bioRxiv.

Jiang F, Taylor DW, Chen JS, Kornfeld JE, Zhou K, Thompson AJ, Nogales E, Doudna JA (2016) Structures of a CRISPR-Cas9 R-loop complex primed for DNA cleavage. Science 351:867-871 Jin S, Zong Y, Gao Q, Zhu Z, Wang Y, Qin P, Liang C, Wang D, Qiu $\mathrm{JL}$, Zhang $\mathrm{F}$ et al (2019) Cytosine, but not adenine, base editors induce genome-wide off-target mutations in rice. Science 364:292-295

Jinek M, Chylinski K, Fonfara I, Hauer M, Doudna JA, Charpentier E (2012) A programmable dual-RNA-guided DNA endonuclease in adaptive bacterial immunity. Science 337:816-821

Kan Y, Ruis B, Takasugi T, Hendrickson EA (2017) Mechanisms of precise genome editing using oligonucleotide donors. Genome Res 27:1099-1111

Kim D, Lim K, Kim ST, Yoon SH, Kim K, Ryu SM, Kim JS (2017a) Genome-wide target specificities of CRISPR RNA-guided programmable deaminases. Nat Biotechnol 35:475-480

Kim K, Ryu SM, Kim ST, Baek G, Kim D, Lim K, Chung E, Kim S, Kim JS (2017b) Highly efficient RNA-guided base editing in mouse embryos. Nat Biotechnol 35:435-437

Kim YB, Komor AC, Levy JM, Packer MS, Zhao KT, Liu DR (2017c) Increasing the genome-targeting scope and precision of base editing with engineered Cas9-cytidine deaminase fusions. Nat Biotechnol 35:371-376

Kim D, Kim DE, Lee G, Cho SI, Kim JS (2019) Genome-wide target specificity of CRISPR RNA-guided adenine base editors. Nat Biotechnol 37:430-435

Klompe SE, Vo PLH, Halpin-Healy TS, Sternberg SH (2019) Transposon-encoded CRISPR-Cas systems direct RNA-guided DNA integration. Nature 571:219-225

Kohama Y, Higo S, Masumura Y, Shiba M, Kondo T, Ishizu T, Higo T, Nakamura S, Kameda S, Tabata T et al (2020) Adeno-associated virus-mediated gene delivery promotes S-phase entry-independent precise targeted integration in cardiomyocytes. Sci Rep 10:15348

Komor AC, Kim YB, Packer MS, Zuris JA, Liu DR (2016) Programmable editing of a target base in genomic DNA without double-stranded DNA cleavage. Nature 533:420-424

Krooss SA, Dai Z, Schmidt F, Rovai A, Fakhiri J, Dhingra A, Yuan Q, Yang T, Balakrishnan A, Steinbruck $L$ et al (2020) Ex vivo/in vivo gene editing in hepatocytes using "All-in-One" CRISPR-AdenoAssociated Virus vectors with a self-linearizing repair template. iScience 23:

Kurt IC, Zhou R, lyer S, Garcia SP, Miller BR, Langner LM, Grunewald J, Joung JK (2020) CRISPR C-to-G base editors for inducing targeted DNA transversions in human cells. Nat Biotechnol 39(1):41-46

Kwart D, Paquet D, Teo S, Tessier-Lavigne M (2017) Precise and efficient scarless genome editing in stem cells using CORRECT. Nat Protoc 12:329-354

Lanza DG, Gaspero A, Lorenzo I, Liao L, Zheng P, Wang Y, Deng Y, Cheng C, Zhang C, Seavitt JR et al (2018) Comparative analysis of single-stranded DNA donors to generate conditional null mouse alleles. BMC Biol 16:69

Lee AY, Lloyd KC (2014) Conditional targeting of Ispd using paired Cas9 nickase and a single DNA template in mice. FEBS Open Bio 4:637-642

Lee K, Mackley VA, Rao A, Chong AT, Dewitt MA, Corn JE, Murthy N (2017) Synthetically modified guide RNA and donor DNA are a versatile platform for CRISPR-Cas9 engineering. Elife 6:

Li H, Beckman KA, Pessino V, Huang B, Weissman JS, Leonetti MD (2019) Design and specificity of long ssDNA donors for CRISPRbased knock-in. BioRxiv.

Liang P, Huang J (2019) Off-target challenge for base editormediated genome editing. Cell Biol Toxicol 35:185-187

Liang $\mathrm{P}, \mathrm{Xu}$ Y, Zhang X, Ding C, Huang R, Zhang Z, Lv J, Xie X, Chen Y, Li Y et al (2015) CRISPR/Cas9-mediated gene editing in human tripronuclear zygotes. Protein Cell 6:363-372

Liang P, Sun H, Sun Y, Zhang X, Xie X, Zhang J, Zhang Z, Chen Y, Ding C, Xiong $Y$ et al (2017) Effective gene editing by high-fidelity base editor 2 in mouse zygotes. Protein Cell 8:601-611

Liang P, Sun H, Zhang X, Xie X, Zhang J, Bai Y, Ouyang X, Zhi S, Xiong $Y$, Ma W et al (2018) Effective and precise adenine base editing in mouse zygotes. Protein Cell 9:808-813

Liang P, Huang JJCB, Toxicology (2019a) Off-target challenge for base editor-mediated genome editing. Cell Biol Toxicol 35:185187

Liang P, Xie X, Zhi S, Sun H, Zhang X, Chen Y, Chen Y, Xiong Y, Ma W, Liu $D$ et al (2019b) Genome-wide profiling of adenine base editor specificity by EndoV-seq. Nat Commun 10:67

Ling X, Xie B, Gao X, Chang L, Zheng W, Chen H, Huang Y, Tan L, Li M, Liu T (2020). Improving the efficiency of precise genome editing with site-specific Cas9-oligonucleotide conjugates. Sci Adv 6:eaaz0051

Liskay RM, Letsou A, Stachelek JL (1987) Homology requirement for efficient gene conversion between duplicated chromosomal sequences in mammalian cells. Genetics 115:161-167

Liu M, Rehman S, Tang X, Gu K, Fan Q, Chen D, Ma W (2018a) Methodologies for improving HDR efficiency. Front Genet 9:691

Liu Z, Chen M, Chen S, Deng J, Song Y, Lai L, Li Z (2018b) Highly efficient RNA-guided base editing in rabbit. Nat Commun 9:2717

Liu Z, Lu Z, Yang G, Huang S, Li G, Feng S, Liu Y, Li J, Yu W, Zhang $Y$ et al (2018c) Efficient generation of mouse models of human diseases via ABE- and BE-mediated base editing. Nat Commun 9:2338

Liu Y, Li X, He S, Huang S, Li C, Chen Y, Liu Z, Huang X, Wang X (2020) Efficient generation of mouse models with the prime editing system. Cell Discov 6:27

Ma Y, Zhang X, Shen B, Lu Y, Chen W, Ma J, Bai L, Huang X, Zhang $L$ (2014) Generating rats with conditional alleles using CRISPR/ Cas9. Cell research 24:122-125

Ma H, Marti-Gutierrez N, Park SW, Wu J, Lee Y, Suzuki K, Koski A, Ji D, Hayama T, Ahmed R et al (2017a) Correction of a pathogenic gene mutation in human embryos. Nature 548:413419

Ma M, Zhuang F, Hu X, Wang B, Wen XZ, Ji JF, Xi JJ (2017b) Efficient generation of mice carrying homozygous double-floxp alleles using the Cas9-Avidin/Biotin-donor DNA system. Cell Res 27:578-581 
Ma H, Marti-Gutierrez N, Park SW, Wu J, Hayama T, Darby H, Van Dyken C, Li Y, Koski A, Liang D et al (2018) Ma et al. reply. Nature 560:E10-E23.

Macintosh KL (2019) Heritable Genome Editing and the Downsides of a Global Moratorium. CRISPR J 2:272-279

Mali P, Yang L, Esvelt KM, Aach J, Guell M, DiCarlo JE, Norville JE, Church GM (2013) RNA-guided human genome engineering via Cas9. Science 339:823-826

Menoret S, De Cian A, Tesson L, Remy S, Usal C, Boule JB, Boix C, Fontaniere S, Creneguy A, Nguyen TH et al (2015) Homologydirected repair in rodent zygotes using Cas9 and TALEN engineered proteins. Sci Rep 5:14410

Miura H, Gurumurthy CB, Sato T, Sato M, Ohtsuka M (2015) CRISPR/Cas9-based generation of knockdown mice by intronic insertion of artificial microRNA using longer single-stranded DNA. Sci Rep 5:12799

Miyasaka Y, Uno Y, Yoshimi K, Kunihiro Y, Yoshimura T, Tanaka T, Ishikubo H, Hiraoka Y, Takemoto N, Tanaka T et al (2018) CLICK: one-step generation of conditional knockout mice. BMC Genomics 19:318

Mohr S, Ghanem E, Smith W, Sheeter D, Qin Y, King O, Polioudakis D, lyer VR, Hunicke-Smith S, Swamy S et al (2013) Thermostable group II intron reverse transcriptase fusion proteins and their use in cDNA synthesis and next-generation RNA sequencing. RNA 19:958-970

Murgha YE, Rouillard JM, Gulari E (2014) Methods for the preparation of large quantities of complex single-stranded oligonucleotide libraries. PLoS ONE 9:

Nakade S, Tsubota T, Sakane Y, Kume S, Sakamoto N, Obara M, Daimon T, Sezutsu H, Yamamoto T, Sakuma T et al (2014) Microhomology-mediated end-joining-dependent integration of donor DNA in cells and animals using TALENs and CRISPR/ Cas9. Nat Commun 5:5560

Nakao H, Harada T, Nakao K, Kiyonari H, Inoue K, Furuta Y, Aiba A (2016) A possible aid in targeted insertion of large DNA elements by CRISPR/Cas in mouse zygotes. Genesis 54:65-77

Ohtsuka M, Sato M, Miura H, Takabayashi S, Matsuyama M, Koyano T, Arifin N, Nakamura S, Wada K, Gurumurthy CB (2018) i-GONAD: a robust method for in situ germline genome engineering using CRISPR nucleases. Genome Biol 19:25

Okamoto S, Amaishi Y, Maki I, Enoki T, Mineno J (2019) Highly efficient genome editing for single-base substitutions using optimized ssODNs with Cas9-RNPs. Sci Rep 9:4811

Paix A, Schmidt H, Seydoux G (2016) Cas9-assisted recombineering in $\mathrm{C}$. elegans: genome editing using in vivo assembly of linear DNAs. Nucleic Acids Res 44:e128

Palermo G, Miao Y, Walker RC, Jinek M, McCammon JA (2016) Striking Plasticity of CRISPR-Cas9 and Key Role of Non-target DNA, as Revealed by Molecular Simulations. ACS Cent Sci 2:756-763

Palermo G, Miao Y, Walker RC, Jinek M, McCammon JA (2017) CRISPR-Cas9 conformational activation as elucidated from enhanced molecular simulations. Proc Natl Acad Sci USA 114:7260-7265

Papaioannou I, Disterer P, Owen JS (2009) Use of internally nuclease-protected single-strand DNA oligonucleotides and silencing of the mismatch repair protein, $\mathrm{MSH} 2$, enhances the replication of corrected cells following gene editing. J Gene Med 11:267-274

Paquet D, Kwart D, Chen A, Sproul A, Jacob S, Teo S, Olsen KM, Gregg A, Noggle S, Tessier-Lavigne M (2016) Efficient introduction of specific homozygous and heterozygous mutations using CRISPR/Cas9. Nature 533:125-129

Pavel-Dinu M, Wiebking V, Dejene BT, Srifa W, Mantri S, Nicolas CE, Lee C, Bao G, Kildebeck EJ, Punjya N et al (2019) Gene correction for SCID-X1 in long-term hematopoietic stem cells. Nat Commun 10:1634

Pritchard CEJ, Kroese LJ, Huijbers IJ (2017) Direct generation of conditional alleles using CRISPR/Cas9 in mouse zygotes. Methods Mol Biol 1642:21-35

Quadros RM, Miura H, Harms DW, Akatsuka H, Sato T, Aida T, Redder R, Richardson GP, Inagaki Y, Sakai D et al (2017) EasiCRISPR: a robust method for one-step generation of mice carrying conditional and insertion alleles using long ssDNA donors and CRISPR ribonucleoproteins. Genome Biol 18:92

Ran FA, Hsu PD, Wright J, Agarwala V, Scott DA, Zhang F (2013) Genome engineering using the CRISPR-Cas9 system. Nat Protoc 8:2281-2308

Rees HA, Wilson C, Doman JL, Liu DR (2019) Analysis and minimization of cellular RNA editing by DNA adenine base editors. Sci Adv 5:eaax5717

Reichmann J, Nijmeijer B, Hossain MJ, Eguren M, Schneider I, Politi AZ, Roberti MJ, Hufnagel L, Hiiragi T, Ellenberg J (2018) Dualspindle formation in zygotes keeps parental genomes apart in early mammalian embryos. Science 361:189-193

Remy S, Chenouard V, Tesson L, Usal C, Menoret S, Brusselle L, Heslan JM, Nguyen TH, Bellien J, Merot J et al (2017) Generation of gene-edited rats by delivery of CRISPR/Cas9 protein and donor DNA into intact zygotes using electroporation. Sci Rep 7:16554

Renaud JB, Boix C, Charpentier M, De Cian A, Cochennec J, Duvernois-Berthet E, Perrouault L, Tesson L, Edouard J, Thinard $R$ et al (2016) Improved genome editing efficiency and flexibility using modified oligonucleotides with TALEN and CRISPR-Cas9 nucleases. Cell Rep 14:2263-2272

Richardson CD, Ray GJ, DeWitt MA, Curie GL, Corn JE (2016) Enhancing homology-directed genome editing by catalytically active and inactive CRISPR-Cas9 using asymmetric donor DNA. Nat Biotechnol 34:339-344

Richardson CD, Kazane KR, Feng SJ, Zelin E, Bray NL, Schafer AJ, Floor SN, Corn JE (2018) CRISPR-Cas9 genome editing in human cells occurs via the Fanconi anemia pathway. Nat Genet 50:1132-1139

Roche PJR, Gytz H, Hussain F, Cameron CJF, Paquette D, Blanchette M, Dostie J, Nagar B, Akavia UD (2018) Doublestranded biotinylated donor enhances homology-directed repair in combination with Cas9 Monoavidin in Mammalian cells. CRISPR J 1:414-430

Rossant J (2018) Gene editing in human development: ethical concerns and practical applications. Development 145

Rouet P, Smih F, Jasin M (1994) Introduction of double-strand breaks into the genome of mouse cells by expression of a rarecutting endonuclease. Mol Cell Biol 14:8096-8106 
Ryu SM, Koo T, Kim K, Lim K, Baek G, Kim ST, Kim HS, Kim DE, Lee $\mathrm{H}$, Chung $\mathrm{E}$ et al (2018) Adenine base editing in mouse embryos and an adult mouse model of Duchenne muscular dystrophy. Nat Biotechnol 36:536-539

Sakuma T, Yamamoto T (2017) Magic wands of CRISPR-lots of choices for gene knock-in. Cell Biol Toxicol 33:501-505

Sather BD, Romano Ibarra GS, Sommer K, Curinga G, Hale M, Khan IF, Singh S, Song Y, Gwiazda K, Sahni J et al (2015) Efficient modification of CCR5 in primary human hematopoietic cells using a megaTAL nuclease and AAV donor template. Sci Transl Med 7:

Savic N, Ringnalda FC, Lindsay H, Berk C, Bargsten K, Li Y, Neri D, Robinson MD, Ciaudo C, Hall J et al (2018) Covalent linkage of the DNA repair template to the CRISPR-Cas9 nuclease enhances homology-directed repair. Elife 7:

Shen B, Zhang X, Du Y, Wang J, Gong J, Zhang X, Tate PH, Li H, Huang X, Zhang W (2013) Efficient knockin mouse generation by ssDNA oligonucleotides and zinc-finger nuclease assisted homologous recombination in zygotes. PLOS ONE 8:

Shen MW, Arbab M, Hsu JY, Worstell D, Culbertson SJ, Krabbe O, Cassa CA, Liu DR, Gifford DK, Sherwood RI (2018) Predictable and precise template-free CRISPR editing of pathogenic variants. Nature 563:646-651

Stahl S, Hultman T, Olsson A, Moks T, Uhlen M (1988) Solid phase DNA sequencing using the biotin-avidin system. Nucleic Acids Res 16:3025-3038

Sternberg SH, LaFrance B, Kaplan M, Doudna JA (2015) Conformational control of DNA target cleavage by CRISPR-Cas9. Nature 527:110-113

Strecker J, Ladha A, Gardner Z, Schmid-Burgk JL, Makarova KS, Koonin EV, Zhang F (2019) RNA-guided DNA insertion with CRISPR-associated transposases. Science 365:48-53

Surun D, Schneider A, Mircetic J, Neumann K, Lansing F, Paszkowski-Rogacz M, Hanchen V, Lee-Kirsch MA, Buchholz F (2020) Efficient generation and correction of mutations in human iPS cells utilizing mRNAs of CRISPR base editors and prime editors. Genes (Basel) 11(5):511

Suzuki K, Tsunekawa Y, Hernandez-Benitez R, Wu J, Zhu J, Kim EJ, Hatanaka F, Yamamoto M, Araoka T, Li Z et al (2016) In vivo genome editing via CRISPR/Cas9 mediated homology-independent targeted integration. Nature 540:144-149

Urnov FD, Rebar EJ, Holmes MC, Zhang HS, Gregory PD (2010) Genome editing with engineered zinc finger nucleases. Nat Rev Genet 11:636-646

Wang J, Exline CM, DeClercq JJ, Llewellyn GN, Hayward SB, Li PW, Shivak DA, Surosky RT, Gregory PD, Holmes MC et al (2015) Homology-driven genome editing in hematopoietic stem and progenitor cells using ZFN mRNA and AAV6 donors. Nat Biotechnol 33:1256-1263

Wang Y, Liu KI, Sutrisnoh NB, Srinivasan H, Zhang J, Li J, Zhang F, Lalith CRJ, Xing H, Shanmugam R et al (2018) Systematic evaluation of CRISPR-Cas systems reveals design principles for genome editing in human cells. Genome Biol 19:62

Wilde JJ, Aida T, Wienisch M, Zhang Q, Qi P, Feng G (2018) Efficient zygotic genome editing via RAD51-enhanced interhomolog repair. bioRxiv.
Wossidlo M, Nakamura T, Lepikhov K, Marques CJ, Zakhartchenko V, Boiani M, Arand J, Nakano T, Reik W, Walter J (2011) 5 -Hydroxymethylcytosine in the mammalian zygote is linked with epigenetic reprogramming. Nat Commun 2:241

Wu Y, Liang D, Wang Y, Bai M, Tang W, Bao S, Yan Z, Li D, Li J (2013) Correction of a genetic disease in mouse via use of CRISPR-Cas9. Cell Stem Cell 13:659-662

Yang H, Wang H, Shivalila CS, Cheng AW, Shi L, Jaenisch R (2013) One-step generation of mice carrying reporter and conditional alleles by CRISPR/Cas-mediated genome engineering. Cell 154:1370-1379

Yang Y, Wang L, Bell P, McMenamin D, He Z, White J, Yu H, Xu C, Morizono H, Musunuru K et al (2016) A dual AAV system enables the Cas9-mediated correction of a metabolic liver disease in newborn mice. Nat Biotechnol 34:334-338

Yang L, Zhang X, Wang L, Yin S, Zhu B, Xie L, Duan Q, Hu H, Zheng $R$, Wei $Y$ et al (2018) Increasing targeting scope of adenosine base editors in mouse and rat embryos through fusion of TadA deaminase with Cas9 variants. Protein Cell 9:814-819

Yao X, Wang X, Hu X, Liu Z, Liu J, Zhou H, Shen X, Wei Y, Huang Z, Ying $W$ et al (2017) Homology-mediated end joining-based targeted integration using CRISPR/Cas9. Cell Res 27:801-814

Yao X, Liu Z, Wang X, Wang Y, Nie YH, Lai L, Sun R, Shi L, Sun Q, Yang H (2018a) Generation of knock-in cynomolgus monkey via CRISPR/Cas9 editing. Cell research 28:379-382

Yao X, Zhang M, Wang X, Ying W, Hu X, Dai P, Meng F, Shi L, Sun Y, Yao $N$ et al (2018b) Tild-CRISPR allows for efficient and precise gene Knockin in mouse and human cells. Dev Cell 45(526-536):

Yeh CD, Richardson CD, Corn JE (2019) Advances in genome editing through control of DNA repair pathways. Nat Cell Biol 21:1468-1478

Yin H, Song CQ, Dorkin JR, Zhu LJ, Li Y, Wu Q, Park A, Yang J, Suresh S, Bizhanova A et al (2016) Therapeutic genome editing by combined viral and non-viral delivery of CRISPR system components in vivo. Nat Biotechnol 34:328-333

Yoon Y, Wang D, Tai PWL, Riley J, Gao G, Rivera-Perez JA (2018) Streamlined ex vivo and in vivo genome editing in mouse embryos using recombinant adeno-associated viruses. Nat Commun 9:412

Yoshimi K, Kaneko T, Voigt B, Mashimo T (2014) Allele-specific genome editing and correction of disease-associated phenotypes in rats using the CRISPR-Cas platform. Nat Commun 5:4240

Yoshimi K, Kunihiro Y, Kaneko T, Nagahora H, Voigt B, Mashimo T (2016) ssODN-mediated knock-in with CRISPR-Cas for large genomic regions in zygotes. Nat Commun 7:10431

Yu Y, Leete TC, Born DA, Young L, Barrera LA, Lee SJ, Rees HA, Ciaramella G, Gaudelli NM (2020) Cytosine base editors with minimized unguided DNA and RNA off-target events and high ontarget activity. Nat Commun 11:2052

Zhang WW, Matlashewski G (2019) Single-strand annealing plays a major role in double-strand DNA break repair following CRISPRCas9 Cleavage in Leishmania. mSphere 4

Zhang JP, Li XL, Li GH, Chen W, Arakaki C, Botimer GD, Baylink D, Zhang L, Wen W, Fu YW et al (2017) Efficient precise knockin with a double cut HDR donor after CRISPR/Cas9-mediated double-stranded DNA cleavage. Genome Biol 18:35 
Zhao D, Li J, Li S, Xin X, Hu M, Price MA, Rosser SJ, Bi C, aZhang X (2020a) Glycosylase base editors enable C-to-A and C-to-G base changes. Nat Biotechnol

Zhao D, Li J, Li S, Xin X, Hu M, Price MA, Rosser SJ, Bi C, Zhang X (2020b) New base editors change $C$ to $A$ in bacteria and $C$ to $G$ in mammalian cells. Nat Biotechnol

Zhou C, Sun Y, Yan R, Liu Y, Zuo E, Gu C, Han L, Wei Y, Hu X, Zeng $R$ et al (2019) Off-target RNA mutation induced by DNA base editing and its elimination by mutagenesis. Nature 571:275-278

Zuccaro MV, Xu J, Mitchell C, Marin D, Zimmerman R, Rana B, Weinstein E, King RT, Palmerola KL, Smith ME et al (2020)
Allele-specific chromosome removal after Cas9 cleavage in human embryos. Cell 183(6):1650-1664

Zuo E, Sun Y, Wei W, Yuan T, Ying W, Sun H, Yuan L, Steinmetz LM, Li Y, Yang H (2019) Cytosine base editor generates substantial off-target single-nucleotide variants in mouse embryos. Science 364:289-292

Zuo E, Sun Y, Yuan T, He B, Zhou C, Ying W, Liu J, Wei W, Zeng R, $\mathrm{Li} Y$ et al (2020) A rationally engineered cytosine base editor retains high on-target activity while reducing both DNA and RNA off-target effects. Nat Methods 17:600-604 